\title{
Cardiac-specific deletion of voltage dependent anion channel 2 leads to dilated cardiomyopathy by altering calcium homeostasis
}

Thirupura S. Shankar (1) 1,2, Dinesh K. A. Ramadurai ${ }^{1}$, Kira Steinhorst ${ }^{3}$, Salah Sommakia ${ }^{1}$, Rachit Badolia1, Aspasia Thodou Krokidi ${ }^{1}$, Dallen Calder ${ }^{1}$, Sutip Navankasattusas ${ }^{1}$, Paulina Sander (1) ${ }^{3}$, Oh Sung Kwon ${ }^{4,5}$, Aishwarya Aravamudhan ${ }^{1}$, Jing Ling ${ }^{1}$, Andreas Dendorfer (10) 6,7, Changmin Xie ${ }^{8}$, Ohyun Kwon (i) ${ }^{8}$, Emily H. Y. Cheng ${ }^{9}$, Kevin J. Whitehead ${ }^{10}$, Thomas Gudermann ${ }^{3,7}$, Russel S. Richardson ${ }^{5}$, Frank B. Sachse ${ }^{1,2}$, Johann Schredelseker (1) ${ }^{3,7}$, Kenneth W. Spitzer ${ }^{1,10}$, Dipayan Chaudhuri (1) ${ }^{1,10}$ \& Stavros G. Drakos (1) ${ }^{1,2,10 凶}$

Voltage dependent anion channel 2 (VDAC2) is an outer mitochondrial membrane porin known to play a significant role in apoptosis and calcium signaling. Abnormalities in calcium homeostasis often leads to electrical and contractile dysfunction and can cause dilated cardiomyopathy and heart failure. However, the specific role of VDAC2 in intracellular calcium dynamics and cardiac function is not well understood. To elucidate the role of VDAC2 in calcium homeostasis, we generated a cardiac ventricular myocyte-specific developmental deletion of Vdac2 in mice. Our results indicate that loss of VDAC2 in the myocardium causes severe impairment in excitation-contraction coupling by altering both intracellular and mitochondrial calcium signaling. We also observed adverse cardiac remodeling which progressed to severe cardiomyopathy and death. Reintroduction of VDAC2 in 6-week-old knockout mice partially rescued the cardiomyopathy phenotype. Activation of VDAC2 by efsevin increased cardiac contractile force in a mouse model of pressure-overload induced heart failure. In conclusion, our findings demonstrate that VDAC2 plays a crucial role in cardiac function by influencing cellular calcium signaling. Through this unique role in cellular calcium dynamics and excitation-contraction coupling VDAC2 emerges as a plausible therapeutic target for heart failure.

\footnotetext{
${ }^{1}$ Nora Eccles Harrison Cardiovascular Research and Training Institute, University of Utah, Salt Lake City, UT, USA. ${ }^{2}$ Department of Biomedical Engineering, University of Utah, Salt Lake City, UT, USA. ${ }^{3}$ Walther Straub Institute of Pharmacology and Toxicology, Faculty of Medicine, LMU Munich, Munich, Germany. ${ }^{4}$ Department of Kinesiology, University of Connecticut, Storrs, CT, USA. ${ }^{5}$ Geriatric Research, Education, and Clinical Center, Salt Lake City VA Medical Center, Salt Lake City, UT, USA. ${ }^{6}$ Walter-Brendel-Center of Experimental Medicine, Ludwig-Maximilians Universität Munich, Munich, Germany. ${ }^{7}$ German Centre for Cardiovascular Research (DZHK), Partner Site Munich Heart Alliance, Munich, Germany. ${ }^{8}$ Department of Chemistry and Biochemistry, University of California, Los Angeles, CA, USA. ${ }^{9}$ Memorial Sloan Kettering Cancer Center, New York, NY, USA. ${ }^{10}$ Division of Cardiovascular Medicine, University of

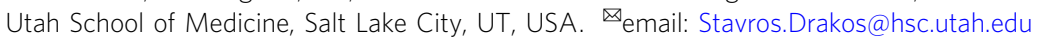


$\mathrm{N}$ on-ischemic dilated cardiomyopathy (DCM) is one of the most common causes that lead to the syndrome of chronic heart failure (HF) which is currently a growing global epidemic ${ }^{1,2}$. DCM is a condition in which the cardiac ventricular chambers enlarge and have impaired systolic and diastolic function. The impaired myocardial function has been partly attributed to alterations in the function of contractile proteins and excitation-contraction coupling (ECC) ${ }^{3}$. Calcium plays a crucial role in ECC and influences cardiac rhythmicity and cellular contraction. During systole, electrical excitation of the membrane causes L-type calcium channels (LTCC) to open and a small amount of calcium enters the cell which binds to ryanodine receptor 2 (RyR2) and triggers the sarcoplasmic reticulum (SR) to release some of its calcium reserve. This release results in an overall increase in the cytosolic calcium which binds to troponin thereby facilitating cellular contraction. The excess cytosolic calcium is pumped out of the cytoplasm through three main processes. Part of the calcium is pumped back into the SR through sarcoplasmic endoplasmic reticulum calcium ATPase 2a (SERCA2a) and the rest of the calcium is extruded out of the cell via the sodium-calcium exchanger (NCX1) and the sarcolemma calcium pump. The mitochondria also appear to play a role in calcium uptake during this process and its importance is under investigation $^{4,5}$.

The voltage-dependent anion channel 2 (VDAC2) is a $32 \mathrm{kDa}$ porin present on the mitochondrial outer membrane (MOM) and contributes to apoptosis, steroidogenesis, metabolite flux, and calcium homeostasis 6 . VDAC2 is known to interact with proapoptotic proteins such as $\mathrm{Bcl} 2$ family proteins. While VDAC2-BAK (Bcl2-antagonist/killer protein) interaction is reported to be highly important to control cellular apoptosis, it is still controversial if this interaction promotes or inhibits apoptosis. Studies show that VDAC2-BAK complex was absent during death stimulus and deletion of VDAC2 promotes apoptosis 7 . In contrast, other studies have shown that truncated-Bid (BH3-interacting domain death agonist) induced MOM permeabilization and cell death in wild-type (WT) but not in VDAC2 knock-out (KO) mouse embryonic fibroblasts ${ }^{8}$.

Calcium flux into the mitochondrial matrix takes place primarily through the mitochondrial calcium uniporter (MCU), present in the mitochondrial inner membrane (MIM). However, the initial calcium import into the inter-membrane space is through VDACs. The interaction between MOM and SR is crucial for this mitochondrial calcium signaling9. Evidence suggests that coupling of VDAC2 with sub-sarcolemmal RyR2 is essential for calcium transfer from SR to the mitochondria ${ }^{10}$. Mitochondrial calcium dynamics have mostly been associated with the MIM and primarily, the MCU. However, it has been reported that MCU-KO mice do not show any adverse cardiac phenotypes ${ }^{11,12}$. This suggests an important role of VDACs and MOM in mitochondrial calcium signaling.

Increasing evidence suggests the importance of VDAC2 in physiologic cardiac function and in this manuscript, we hypothesize that mitochondrial calcium dynamics have a significant influence in this process ${ }^{13,14}$. Increasing the calcium uptake through VDAC2 has been shown to reverse the arrhythmia phenotype observed in the tremblor zebrafish model (NCX1h mutant) and the tachycardia phenotype in mice with catecholaminergic polymorphic ventricular tachycardia (RYR2 mutant $)^{13,14}$. Both these arrhythmic models have irregular cellular calcium signaling. Enhancing mitochondrial calcium uptake through VDAC2 using a small molecule called efsevin, pharmacologically helped restore the rhythmic phenotype in these models. VDAC2 knock-down in HL-1 cell line has shown to have specific effects on calcium homeostasis such as increased diastolic calcium and restricted calcium spark expansion ${ }^{15}$. However, after a global knockdown of VDAC2 was shown to be embryonic lethal ${ }^{7}$, no further studies have investigated the effects of cardiac VDAC2-KO in animal models. Advancing our understanding of VDAC2's role in calcium signaling and ECC may have significant implications. In this study, in an effort to address this gap in knowledge we performed cardiac-specific Vdac2 deletion and studied its effects on calcium cycling. Our results indicate a strong dependency on VDAC2 and cellular calcium dynamics to the ECC. VDAC2-KO mice showed significantly slower mitochondrial calcium uptake and altered cellular calcium signaling with smaller calcium transient, a slower rate of decay, and a slower rate of rising in cytosolic calcium. Adverse cardiac remodeling and severe cardiomyopathy led to the death of these $\mathrm{KO}$ mice. Reintroduction of VDAC2 in 6-week-old $\mathrm{KO}$ mice using an adeno-associated virus 9 (AAV9) vector seemed to partially rescue the cardiomyopathy phenotype suggesting a plausible role of VDAC2 as a therapeutic target in clinical HF. To evaluate this hypothesis, we took a gain-of-function approach and measured the effects of VDAC2 agonist efsevin in murine tissue from failing hearts: in line with the HF phenotype induced by VDAC2-KO, efsevin enhanced contractile force in failing myocardium from a murine pressure-overload model establishing VDAC2 as a promising target for $\mathrm{HF}$.

\section{Results}

Cardiac-specific developmental deletion of VDAC2 causes DCM and leads to increased mortality. Cardiac ventriclespecific developmental Vdac2 $\mathrm{KO}$ and littermate WT were used for this study. Mice with either flox or Cre were used as WT and echocardiography data on flox or Cre only mice shows no significant differences (Supplementary Fig. 1a-g). Deletion of VDAC2 was confirmed using western blot (Fig. 1a). We observed no difference in VDAC1 and VDAC3 protein levels in VDAC2KO mice (Supplementary Fig. 2a-c), suggesting a pure VDAC2dependent phenotype. Increased mortality was observed in the KO between 5 and 20-weeks postpartum. Most of these events occurred during 20- weeks postpartum (Fig. 1b). Serial echocardiographic analysis, showed progressive deterioration of cardiac function in VDAC2-KO mice as reflected by significant reductions around 16 weeks in left ventricular (LV) ejection fraction (EF), fractional shortening (FS), and posterior wall thickness at systole (LVPW,s) and a significant increase in LV end-diastolic diameter (LVEDD), LV end-diastolic and endsystolic volumes (EDV and ESV, respectively). No difference in LV posterior wall thickness at diastole (LVPW,d) and LV mass (normalized to body weight) was observed (Fig. $1 \mathrm{c}-\mathrm{j}$ ). We are also providing Supplementary Table 1 which describes the cardiac phenotype during the entire development, including intra-utero echocardiograms performed in pregnant mice ${ }^{16}$. We also found increased gene expression of NPPA in KO mice (Fig. $1 \mathrm{~m}$ ), all of which are hallmarks of DCM. Increased dilation was also evident from echocardiography and the $\mathrm{KO}$ hearts were significantly larger than WT hearts (Fig. 1k, l). VDAC2-KO mice also had significantly longer and thinner cardiomyocytes compared to WT mice at 16-weeks of age (Fig. $1 \mathrm{n}-\mathrm{p}$ ). Taken together, our results suggest that deletion of VDAC2 in the myocardium during development causes DCM and eventually leads to increased mortality.

Altered gene and protein expression profiles indicate altered calcium signaling and regulation in VDAC2-KO. Gene expression profile from ventricular RNA of 16-week-old KO mice showed a number of differentially expressed genes involved in calcium signaling and regulation. Calcium channel genes such as Cacna1h, a T-type calcium channel (Cav 3.2) was upregulated 
a
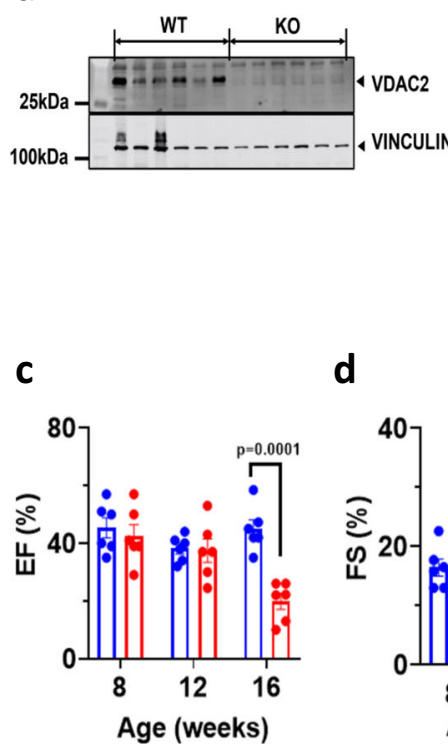

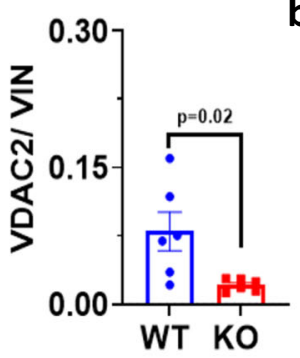

d

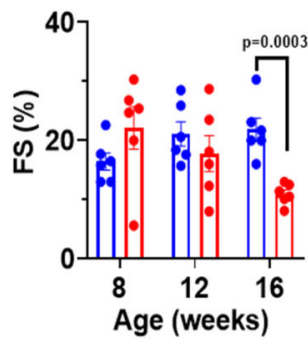

e b

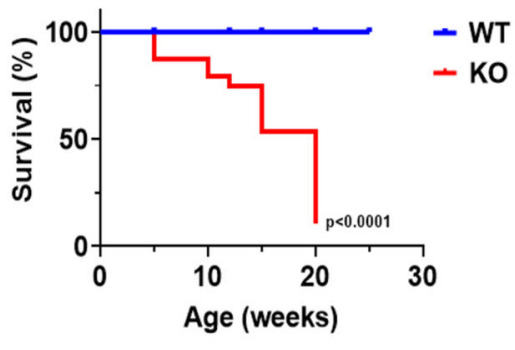

f
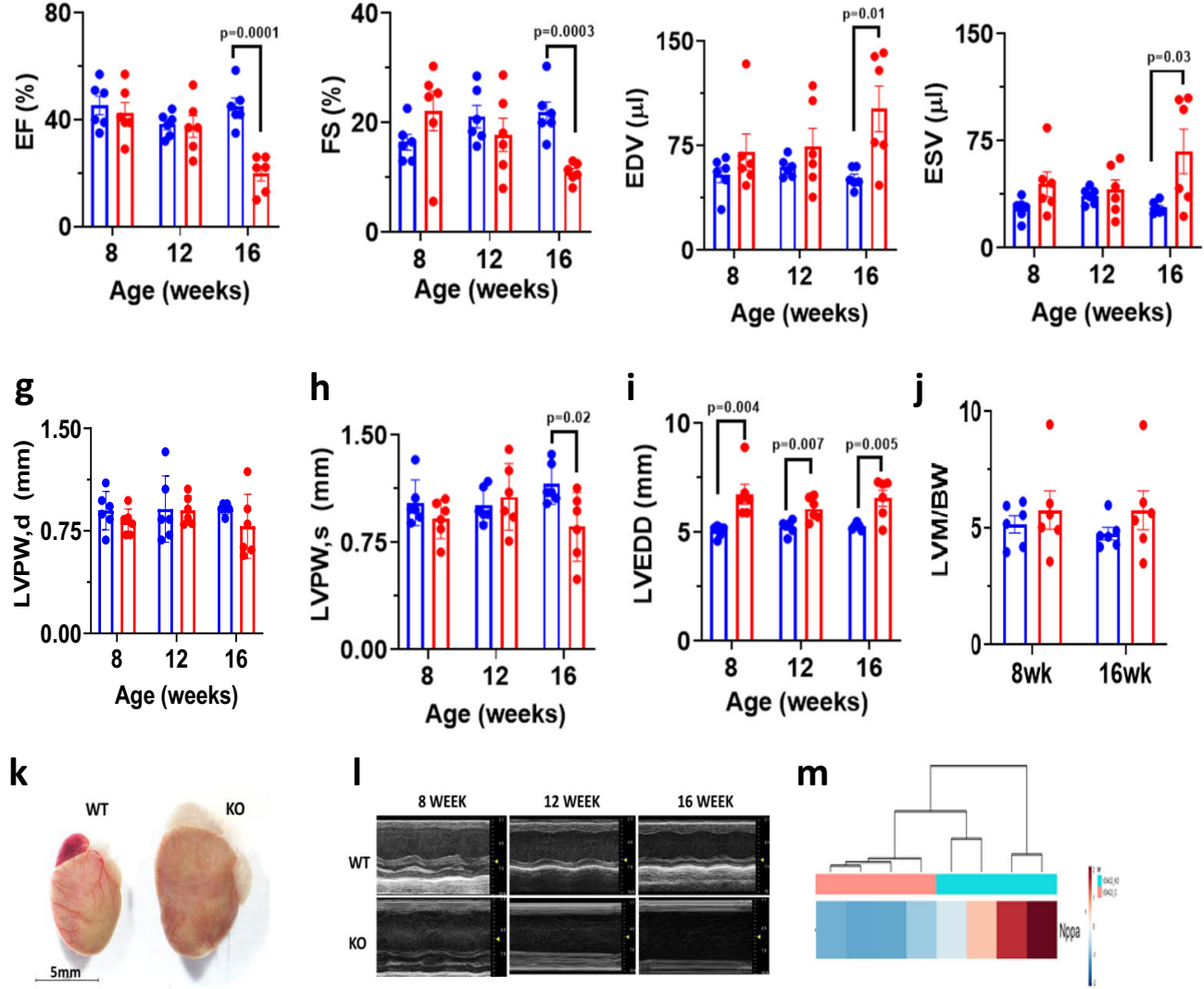

n

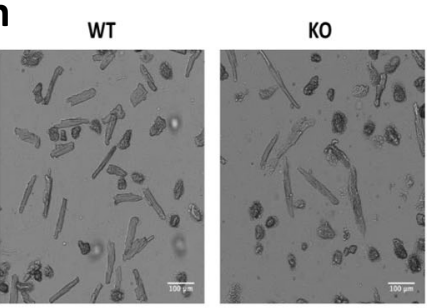

o
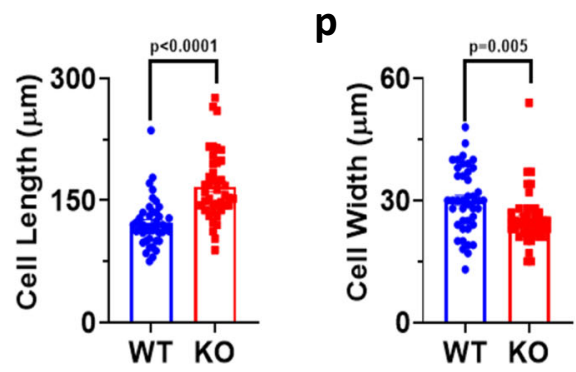

Fig. 1 Cardiac-specific deletion of VDAC2 causes dilated cardiomyopathy and leads to increased mortality. a Western blot image of VDAC2 and laneloading control VINCULIN and quantification in WT (Wild-type) and KO (Knock-out) using Image Studio Lite (version5.2.5) $(p=0.02)(n=6)$;

b Kaplan-Meier survival curve $(n=10)(p<0.0001)$; $\mathbf{c}-\mathbf{j}$ Echocardiography analysis of left ventricular ejection fraction (EF) ( $p=0.000143)$, fractional shortening (FS) (0.000376), left ventricular end-diastolic and end-systolic volumes (EDV and ESV) ( $p=0.0132$ and $p=0.0311$ ), left-ventricular posterior wall thickness at diastole and systole (LVPW, $\mathrm{d}$ and LVPW, $\mathrm{s})(p=\mathrm{ns}$ and $p=0.0244)$, left ventricular end-diastolic diameter (LVEDD) (8-week $p=$ $0.004824 ; 12$-week $p=0.007670$ and 16-week $p=0.005986)(n=6)$ and $\mathbf{j}$ left ventricular mass to body weight ratio (LVM/BW), respectively ( $n=6)$; k Representative heart image of 16-week-old mice (scale bar, $5 \mathrm{~mm}$ ); I Representative m-mode image from echocardiography $(n=1) ; \mathbf{m}$ Differential expression of NPPA gene $(n=4) ; \mathbf{n}$ Representative ventricular cardiomyocyte image using confocal microscopy (scale bar, $100 \mu \mathrm{m}$ ); $\mathbf{0}, \mathbf{p}$ Cardiomyocyte cell length $(p<0.0001)$ and width $(p=0.0058)$ measured using Zen5 imaging software, respectively $(n=42, N=3)$. $p$-value: unpaired two-tailed $t$-test performed in panels $\mathbf{a}, \mathbf{o}$, and $\mathbf{p}$. Mantel-Cox test performed in panel $\mathbf{b}$ and two-stage set-up multiple $t$-test (Benjamini, Krieger, and Yekutieli) performed for longitudinal echocardiographic data. Data are represented as differences between mean \pm SEM. 
a

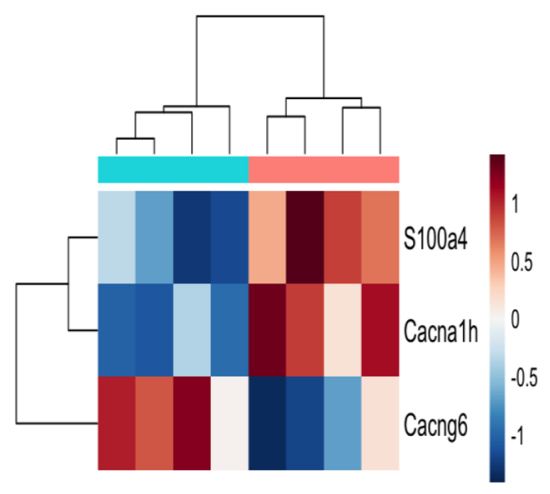

b

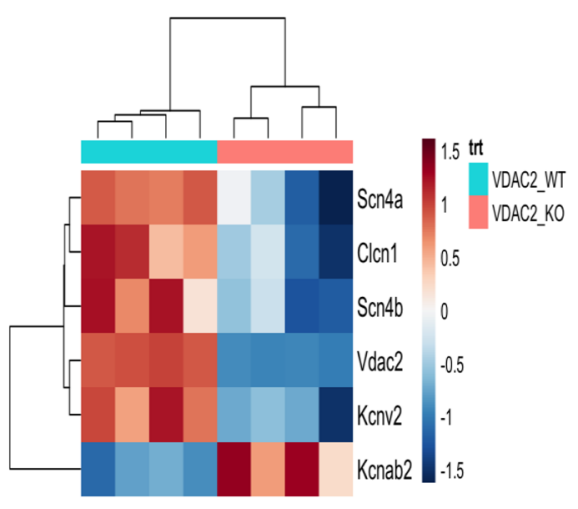

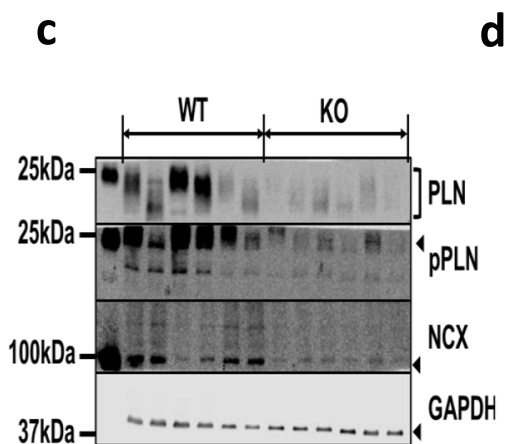

d e

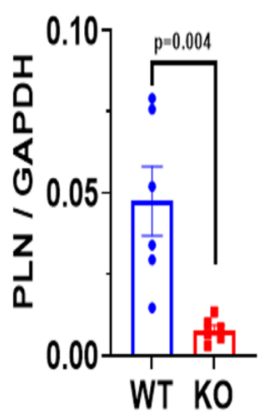

e

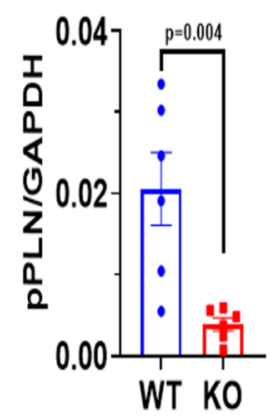

f

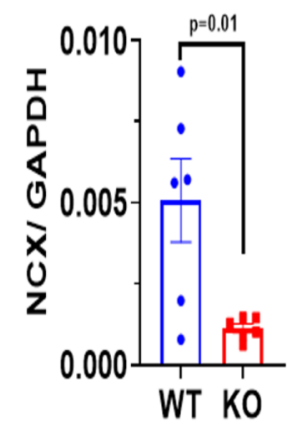

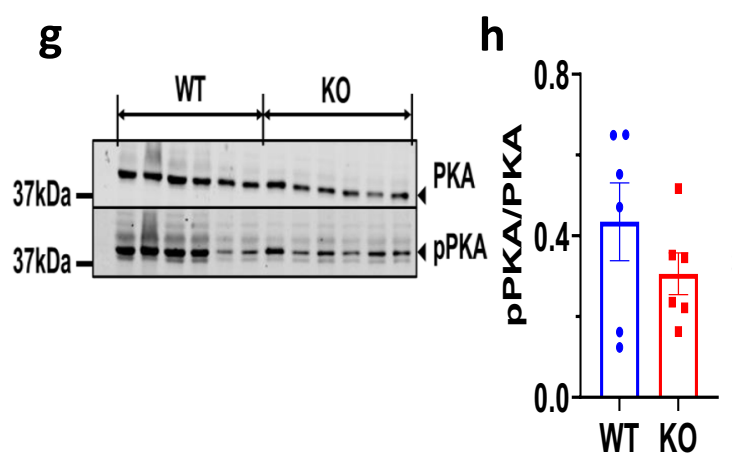

i

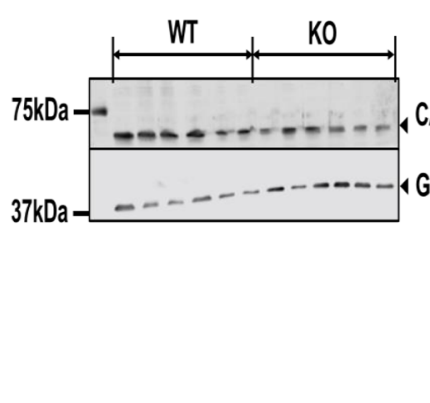

j

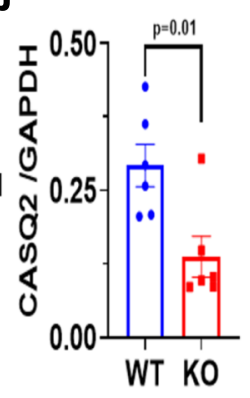

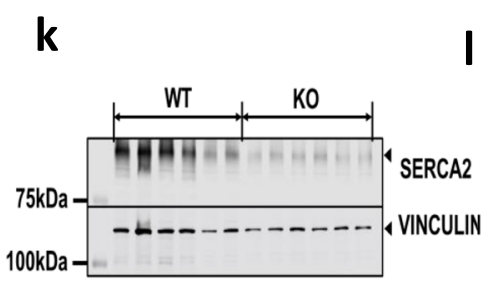

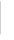

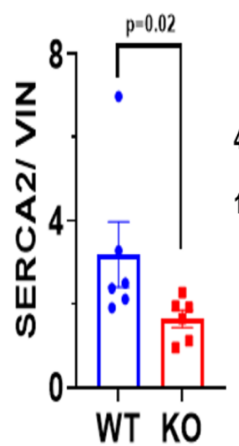

m

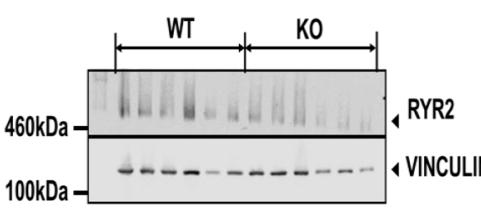

n

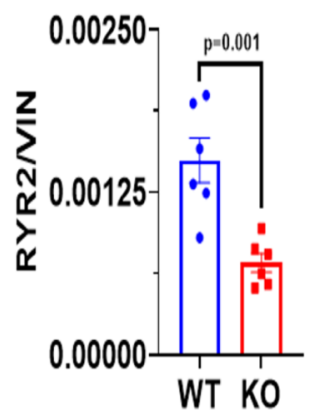

whereas Cacng6, a subunit of the LTCC was significantly downregulated (Fig. 2a). Other ion channels were also differentially expressed between the two groups. Specifically, a significant downregulation of sodium and potassium ion channels was observed in VDAC2-KO mice, including $S c n 4 a, S c n 4 b$, and $K c n v 2$, all of which have been reported to play significant roles in normal cardiac function (Fig. 2b). Proteins involved in ECC such as RYR2, SERCA2, NCX1, total and phosphorylated phospholamban (PLN), and calsequestrin (CASQ2, the SR calciumbinding protein) were significantly decreased in the $\mathrm{KO}$ (Fig. 2c-n). Collectively, these results suggest that loss of VDAC2 leads to abnormalities in cellular calcium signaling pathways. 
Fig. 2 Altered gene and protein expression profiles indicate altered calcium signaling and regulation in VDAC2-KO. a, b Differential gene expression data derived from 16-week old cardiac ventricular tissue $(n=4)$; c-f Western blot image of PLN $(p=0.0042), \operatorname{pPLN}(p=0.0045)$, and NCX $(p=0.0121)$ along with lane-loading control GAPDH and respective quantification using Image Studio Lite (version 5.2.5) $(n=6) ; \mathbf{g}$, $\mathbf{h}$ Western blot image of PKA and pPKA and quantification using Image Studio Lite (version 5.2.5) ( $p=n s)(n=6) ; \mathbf{i}, \mathbf{j}$ Western blot image of CASQ2 and lane-loading control GAPDH and respective quantification using Image Studio Lite (version 5.2 .5$)(p=0.0111)(n=6) ; \mathbf{k}$, I Western blot image of SERCA2 and lane-loading control VINCULIN and quantification using Image Studio Lite (version 5.2.5) (Mann-Whitney: $p=0.0260)(n=6) ; \mathbf{m}, \mathbf{n}$ Western blot image of RYR2 and laneloading control VINCULIN and quantification using Image Studio Lite (version 5.2.5) $(p=0.0018)(n=6)$. $p$-value: unpaired two-tailed $t$-test and twotailed Mann-Whitney test performed. Data are represented as mean \pm SEM.

a
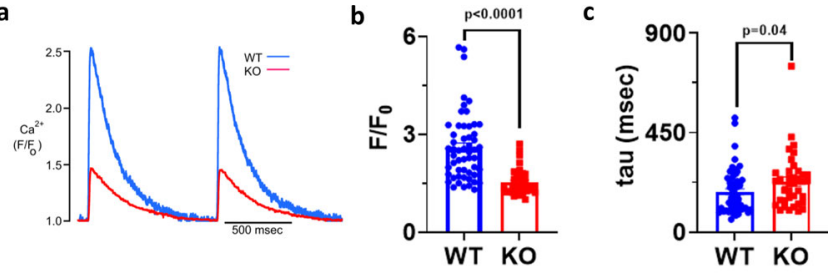

d

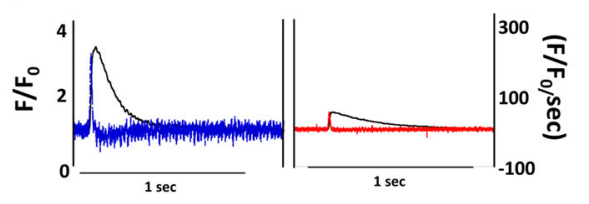

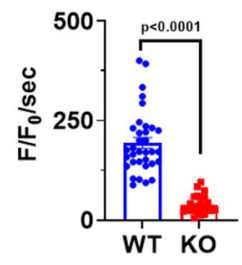

Fig. 3 Impaired calcium cycling in VDAC2-KO cardiomyocytes. a

Representative calcium transient image of 16-week-old mice (Time frame, $500 \mathrm{~ms}$ ); b Amplitude $\left(F / F_{0}\right)$ data ( $p<0.0001$ ) (WT $n=54, \mathrm{KO} n=38$, $N=3$ ); c Rate of calcium uptake (tau) (ms) ( $p=0.0492)$ (WT $n=54$, KO $n=38, N=3)$; d Representative image of rate of calcium release of 16week-old mice (Time frame, $1 \mathrm{~s})$; e Rate of calcium release $\left(F / F_{0} / s\right)(p<$ 0.0001) (WT $n=34, \mathrm{KO} n=23, N=3$ ). $p$-value: unpaired two-tailed $t$-test performed in all comparisons. Data are represented as mean $\pm \mathrm{SEM}$.

Impaired calcium cycling in VDAC2-KO cardiac myocytes. To further assess calcium handling in these mice, we recorded calcium transients in 16-week-old cardiac myocytes. Compared to WT, VDAC2-KO myocytes had smaller calcium transients $\left(F / F_{0}\right)$ with a slower rate of decay (tau) and slower maximum rate of rising $\left(F / F_{0} / \mathrm{s}\right)$ (Fig. 3a-e). To investigate if the decreased calcium transients are directly attributable to the lack of VDAC2 we tested the effects of VDAC2 agonist efsevin ${ }^{14}$ on calcium transients in WT cardiomyocytes and found the opposite effect, namely an increase in calcium transient amplitude and an acceleration of the decay phase (Supplementary Fig. 3a-c). SERCA2a and NCX1 are the two main channels involved in cytosolic calcium clearance during diastole and we showed that these protein levels are significantly reduced in VDAC2-KO (Fig. 2f and 1). Coimmunoprecipitation (Co-IP) studies using whole-heart lysate and pull-down using VDAC2 antibody revealed VDAC2-SERCA2 interaction and VDAC2-NCX1 interaction, respectively. VDAC2-KO heart was used as a negative control for co-IP (Fig. 4a). This interaction was also confirmed by immunofluorescence and proximity ligation assays (PLA) (Fig. 4b, c). We hypothesize that these interactions, similar to the previously discovered VDAC2-RyR2 interaction ${ }^{10}$, are crucial for calcium uptake by the mitochondria and indirectly by the SR. As a result, a disruption of this interaction caused drastic changes in the calcium signaling in the cardiac myocytes. This altered calcium signaling may explain bradyarrhythmia observed in VDAC2-KO (Fig. 5a, b) ${ }^{17}$. Additionally, in agreement with the altered gene expression of the above-mentioned ion channels, the action

potential (AP) of the $\mathrm{KO}$ was different from that of WT with a significantly longer $\mathrm{AP}$ duration at $50 \%$ repolarization $\left(\mathrm{APD}_{50}\right)$ and a normal $90 \%$ repolarization $\left(\mathrm{APD}_{90}\right)$ (Fig. $\left.5 \mathrm{c}-\mathrm{e}\right)$. Collectively, we found significant differences in most aspects of calcium cycling in the VDAC2-KO, which presumably contributes to the progressive decline in myocardial function.

Altered mitochondrial structure and function in VDAC2-KO. Transmission electron microscopy (TEM) performed on 16week-old KO and WT hearts showed substantial alterations in mitochondrial distribution and structure (Fig. 6a-c). KO mitochondria were significantly smaller, disoriented, and less dense compared to WT mitochondria. We did not observe any significant difference in dynamin-related protein 1 (DRP1) levels (a marker for mitochondrial fission) although it showed a trend towards an increase in $\mathrm{KO}$ mice (Fig. 6d, e). We also observed a significant reduction in the rate of calcium uptake in $\mathrm{KO}$ mitochondria suggesting VDAC2 plays an important role in mitochondrial calcium transport (Fig. 6f-h). A significant decline in MCU and NCLX protein levels was also observed in the KO (Fig. 6i-k). These proteins are known to play an important role in mitochondrial calcium transport and signaling and the decreased protein level in the $\mathrm{KO}$ is consistent with the slower rate of calcium uptake.

Increased cardiac fibrosis observed in VDAC2-KO. Gene expression profile showed a significant upregulation of extracellular matrix (ECM) proteins and collagen encoding genes including Col3a1, Col5a2, and Mmp12 in the KO that suggests increased collagen deposition and fibrosis (Fig. 7a). Kyoto Encyclopedia of Genes and Genomes (KEGG) pathway analysis in KO mice indicated that the ECM production pathway changes reached the highest statistical significance (Fig. 7b). Masson's trichrome stain and TEM on 16-week-old mice hearts showed significantly higher collagen content in the KO (Fig. 7c-f). The observed increased myocardial fibrosis is consistent with adverse structural and functional myocardial remodeling and cardiomyopathy in the $\mathrm{KO}$ mice.

Metabolic alterations observed in VDAC2-KO. Since VDAC2 is known to interact with a number of key enzymes like phosphofructokinase (PFK) and hexokinase (HK) that are involved in glycolysis and is known to transport a number of metabolites and ATP, we also measured the RNA and protein levels of specific enzymes in key metabolic pathways. The volcano plot from RNA sequencing data showed significant differences in enzymes involved in metabolism and we observed an overall downregulation of enzyme levels involved in glycolysis, tricarboxylic acid (TCA) cycle, and glycolytic accessory pathways. Specifically, enzymes including transketolase (TKT), succinyl-CoA ligase (SUCLA2), pyruvate dehydrogenase $(\mathrm{PDH})$, and pyruvate carboxylase (PC). However, no significant difference was observed in fatty acid oxidation (FAO) pathways (Supplementary Fig. 4a-o). VINCULIN and GAPDH were used as lane-loading controls and 
a

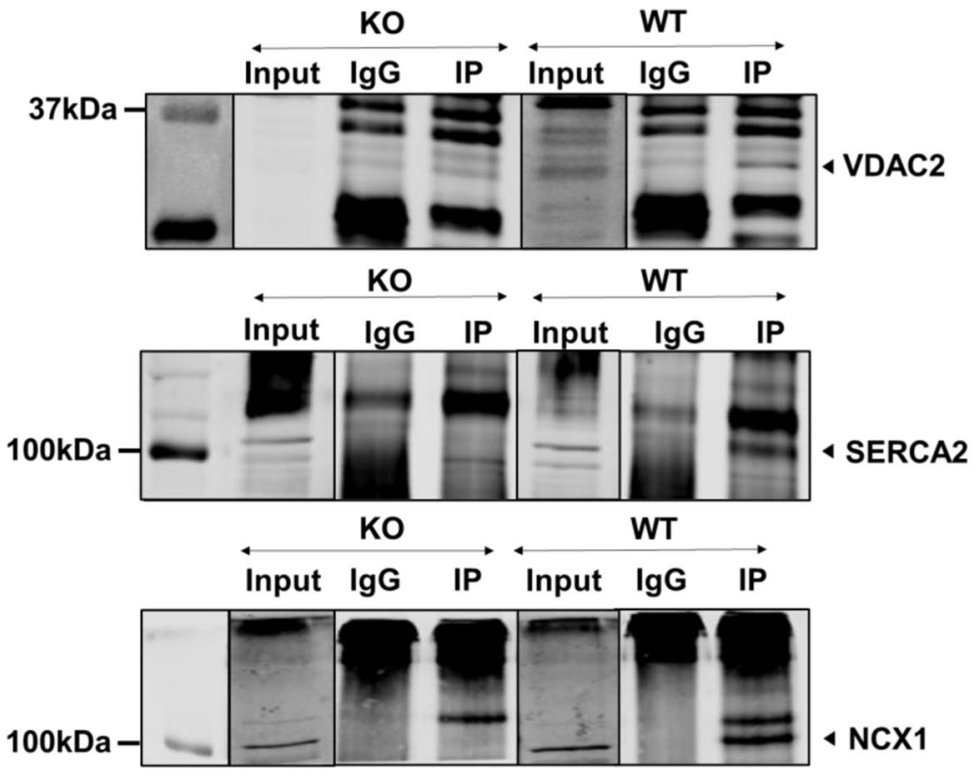

b

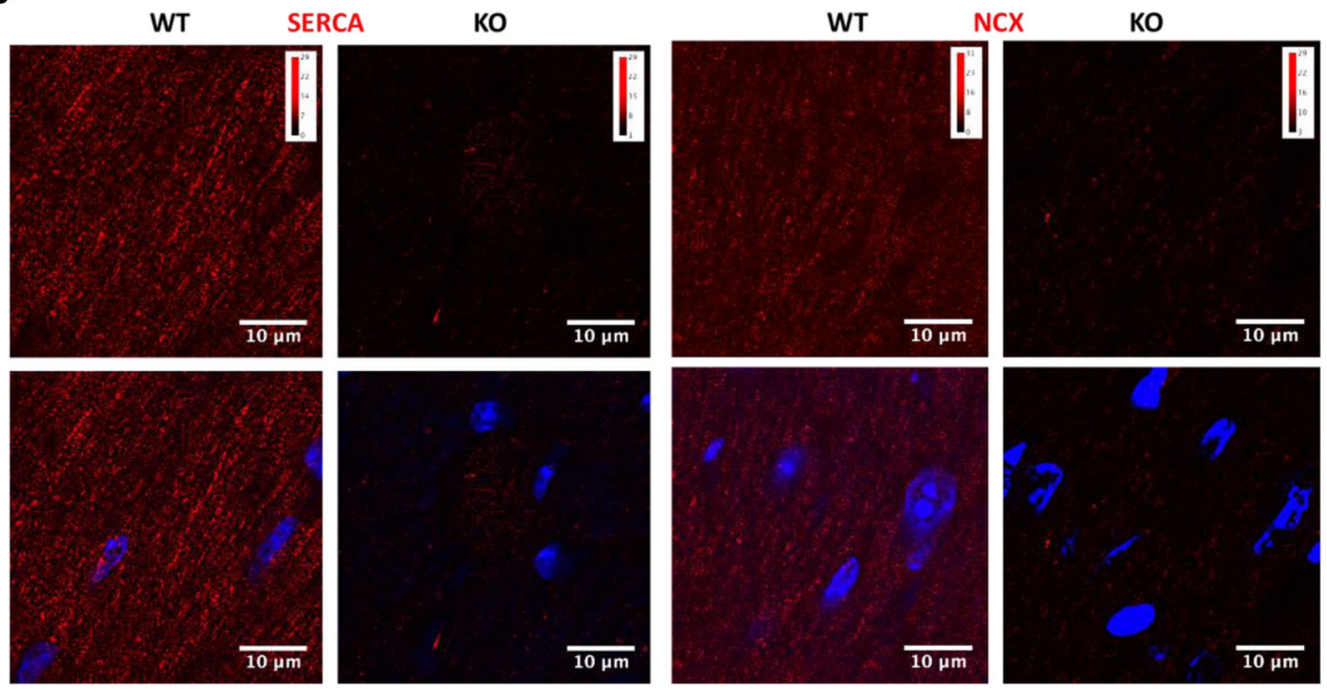

C
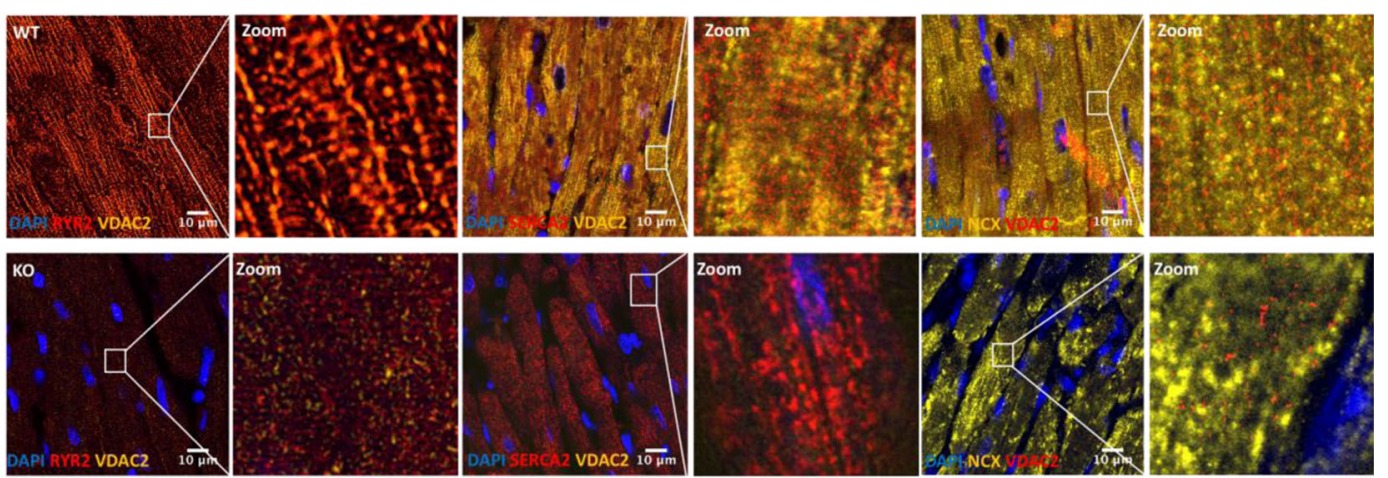

Fig. 4 Cross-talk between mitochondria and ER/SR facilitates mitochondrial calcium cycling. a Representative co-immunoprecipitation image from a cardiac ventricular sample of 8 -week-old mice $(n=3)$ in WT and KO (Input-non-IP sample; IgG-control antibody; IP-VDAC2 protein pull-down); b Representative proximity ligation assay image for VDAC2-SERCA2 and VDAC2-NCX1 interaction in 8-week-old mice (scale bar, $10 \mu \mathrm{m})(n=3)$; c Representative immunofluorescence image from 16-week-old mice heart (scale bar, $10 \mu \mathrm{m})(n=3)$. 


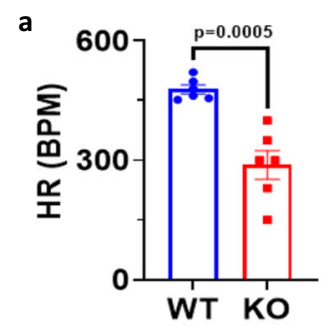

b

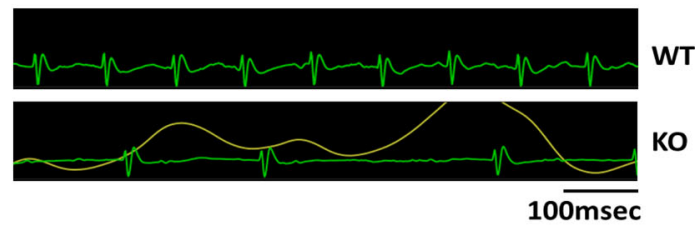

C

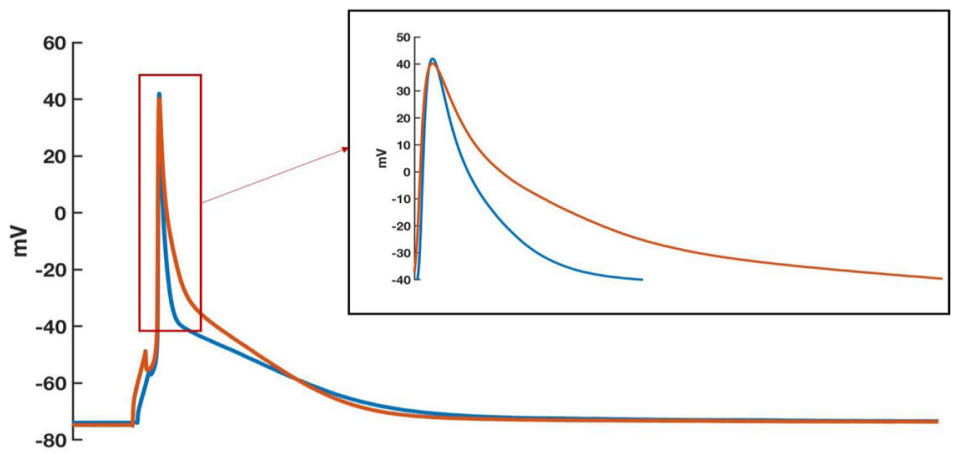

d

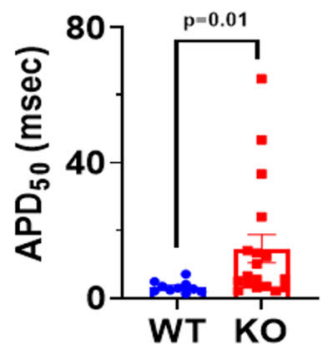

e

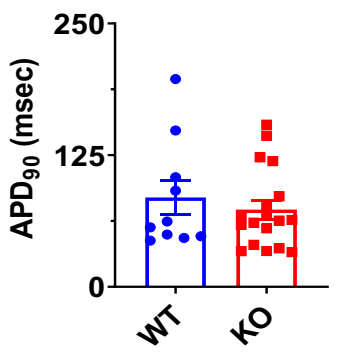

Fig. 5 Altered action potential in VDAC2-KO cardiomyocytes. a Heart rate (HR), beats per minute (BPM) $(p=0.0005)(n=6)$; b Representative ECG of 16-week-old mice (time frame, $100 \mathrm{~ms}$ ); c Representative action potential image of 16-week-old mice cardiomyocyte (time frame, $100 \mathrm{ms);} \mathrm{d,} \mathrm{e} \mathrm{Action}$ potential repolarization duration at $50 \%$ and $90 \%$, respectively (two-tailed Welch test: APD90 $p=n s, \operatorname{APD} 50 p=0.0148)(W T n=10, K O n=18, N=3)$. $p$-value: unpaired two-tailed $t$-test and two-tailed Welch test performed. Data are represented as mean \pm SEM.

we observed no difference in these protein levels between WT and KO (Supplementary Fig. 4p).

No significant difference in mitochondrial ROS production. VDAC2-KO did not show a significant difference (compared to WT) in reactive oxygen species (ROS) production upon inhibition of complex I and complex III using rotenone and antimycin, respectively (Supplementary Fig. 5a-d). However, we found a trend $(p=0.08)$ towards a decrease in complex II in the $\mathrm{KO}$ (Supplementary Fig. 5e-i).

Unaltered mitochondrial respiration. Oxidative phosphorylation (OXPHOS) measurements with LV myocardial tissue showed no difference in mitochondrial oxygen consumption between the WT and KO under normal conditions (Supplementary Fig. 6a-i). This suggests that VDAC2 is not essential for normal mitochondrial respiration despite its role in calcium signaling. These results are similar to those observed in previous publications with MCU-KO ${ }^{11,18}$.

Partial rescue of HF phenotype upon VDAC2 re-introduction. Cardiac ventricular myocyte-specific reintroduction of VDAC2 in $\mathrm{KO}$ and WT mice was assessed using western blot and qRT-PCR
(Fig. 8a, b). Serial echocardiography was performed on these mice up to 10 weeks post-injection (16-week-old mice) to get comparable results. Cardiac structural and functional improvement was observed in $\mathrm{KO}$ mice injected with AAV9-aMHC-VDAC2GFP vector evident from relative improvement in EF, FS, and LV volumes and from a decrease in LV diameter compared to $\mathrm{KO}$ mice injected with control AAV9-aMHC-GFP vector (Fig. 8c-g). Complete rescue of the phenotype was not observed, however, the re-introduction of VDAC2 aided in better cardiac functioning.

VDAC2 as a pharmacological target for HF. We show that VDAC2 regulates cardiac calcium signaling without having major effects on bioenergetics and VDAC2 deletion causes DCM, making it a candidate structure for HF therapy. We therefore, addressed the question of increasing VDAC2s calcium uptake activity could enhance cardiac contractile force in HF. Therefore, we used the established model of pressure overload-induced HF by transverse aortic constriction (TAC). Since the administration of efsevin to mice is difficult for more than a few days due to its short plasma half-life time and efsevin is not orally bioavailable, we took advantage of organotypic heart slices and investigated the effect of efsevin on myocardial contractile strength. Strikingly, efsevin enhanced contractile strength and accelerated relaxation in both control and failing hearts (Fig. 9a, b). 
a

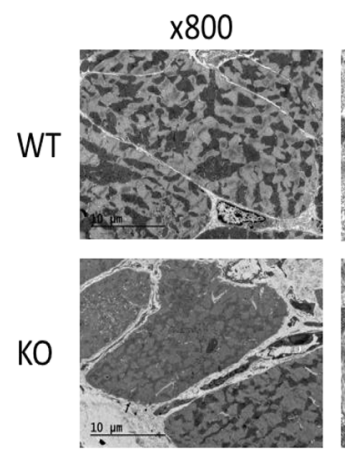

C

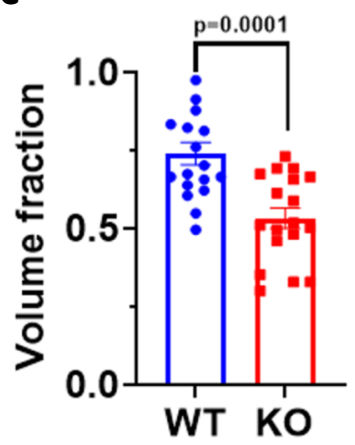

f

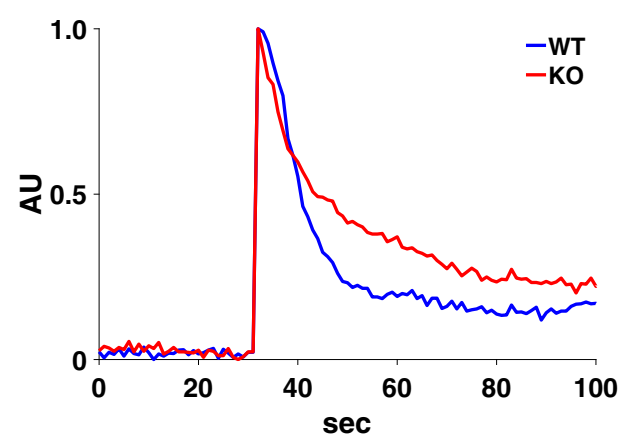

i

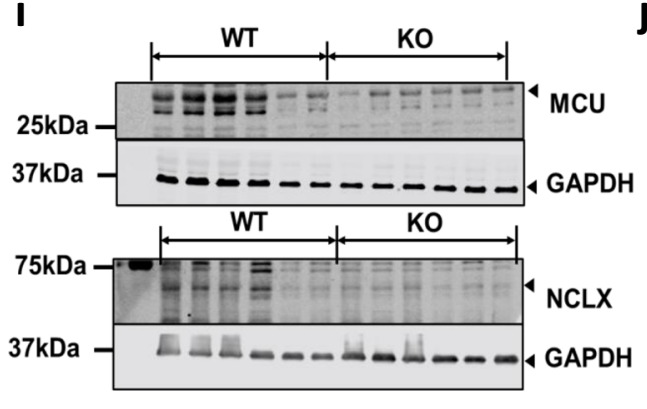

b

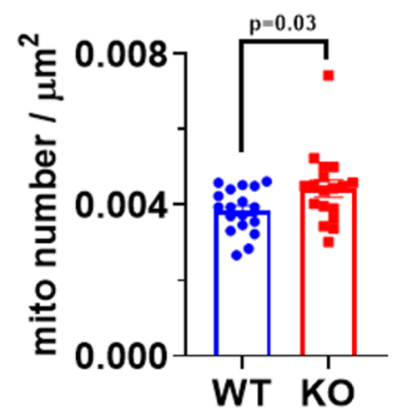

e

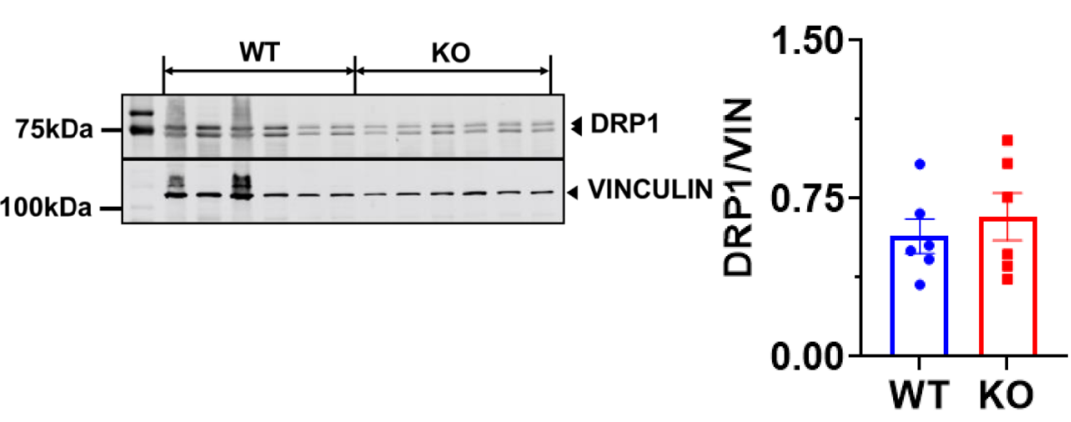

g

h

30 SECONDS
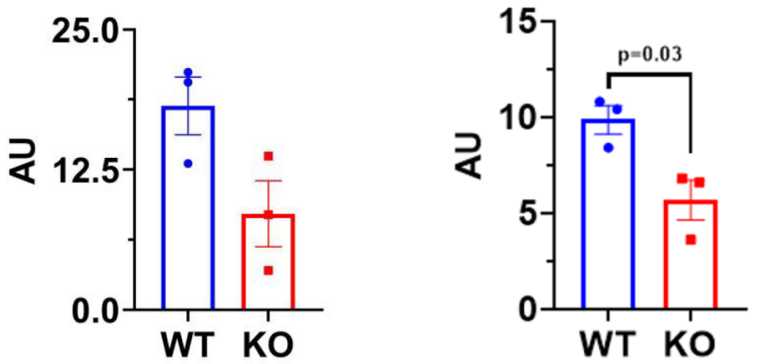

k

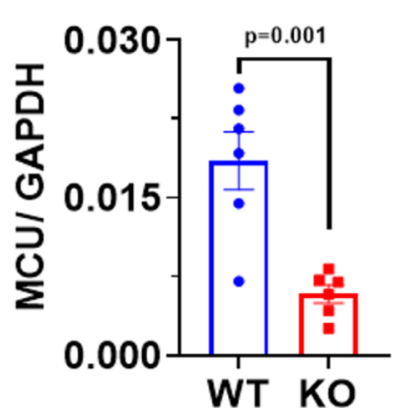

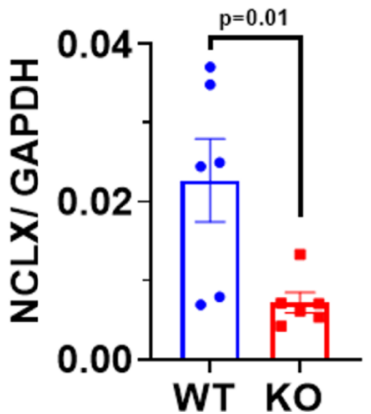

\section{Discussion}

In this study, we investigated the role of VDAC2 in the heart by creating a cardiac ventricular myocyte-specific deletion of VDAC2 using $\alpha$-Myosin heavy chain ( $\mathrm{aMHC}$ ) promoter. We observed increased postnatal mortality along with severe impairment in cardiac structure and function consistent with
DCM. Our gene and protein studies suggested altered calcium signaling in the $\mathrm{KO}$ mice. In agreement with these data, the $\mathrm{KO}$ showed smaller calcium transients, a slower decay time, and a slower rate of calcium release. Substantial adverse cardiac remodeling and mitochondrial disorganization along with slower mitochondrial calcium uptake were also observed in the $\mathrm{KO}$ 
Fig. 6 Altered mitochondrial structure and function in VDAC2-KO. a Representative transmission electron microscopy image at $\times 800, \times 5000$ and $\times 15,000$ magnifications (scale bar, $10 \mu \mathrm{m}, 1 \mu \mathrm{m}$, and $500 \mathrm{~nm}$, respectively) ( $n=3$ ); b Number of mitochondria per unit area measured using Adobe Photoshop CC 2019 ( $p=0.0365)$ ( $n=18$ images, $N=3$ ); c Mitochondrial volume fraction measured using Adobe Photoshop CC 2019 using $22 \times 14$ grid $(p=0.0001)(n=18$ images, $N=3)$; d, e Western blot image of DRP1 and lane-loading control VINCULIN and quantification using Image Studio Lite (version 5.2.5) ( $p=n s)(n=6) ; \mathbf{f}$ Representative mitochondrial calcium uptake image produced using MATLAB $(n=3) ; \mathbf{g}$, $\mathbf{h}$ Mitochondrial calcium uptake in the first $30 \mathrm{~s}(p=n s)$ and $60 \mathrm{~s}$ of calcium pulse $(p=0.0300)(n=3)$, respectively; i-k Western blot image of MCU $(p=0.0014)$ and NCLX $(p=$ 0.0169) along with respective lane-loading control GAPDH and quantification using Image Studio Lite (version 5.2.5) $(n=6)$. $p$-value: unpaired two-tailed $t$-test performed in all comparisons. Data are represented as \pm SEM.

a

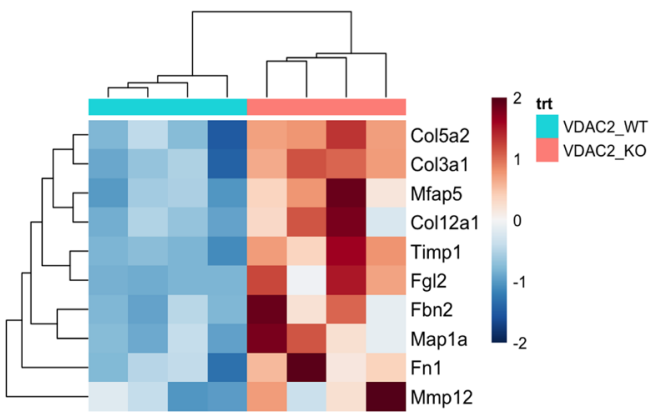

C

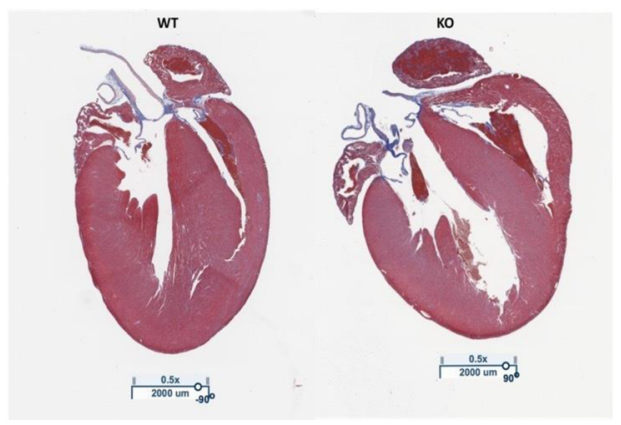

e

WT

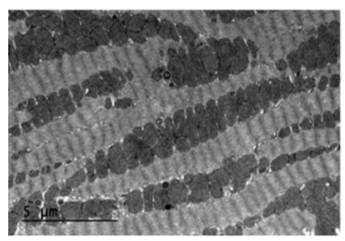

b

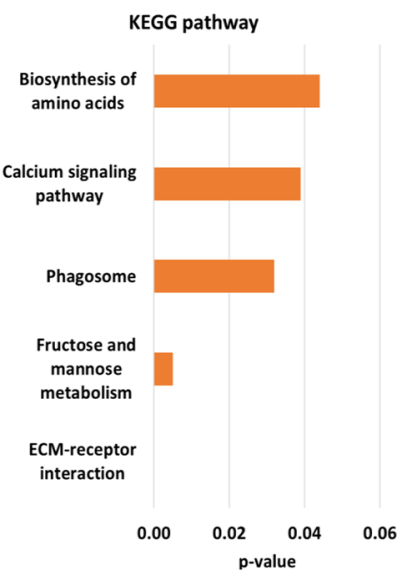

d

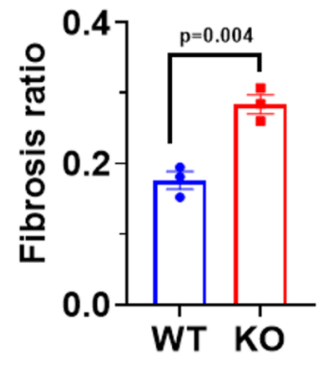

$\mathbf{f}$

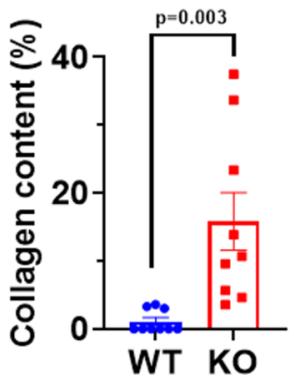

Fig. 7 Increased cardiac fibrosis observed in VDAC2-KO. a Differential gene expression data from 16-week-old mice ventricular sample ( $n=4)$; b KEGG pathway analysis of differentially expressed genes in KO indicates ECM production pathway as highest statistical significance $(n=4)$; $\mathbf{c}$ Representative Masson's trichrome stain image $(\times 0.5$ magnification; scale bar, $2000 \mu \mathrm{m})(n=3)$; d Fibrosis quantification using Aperio software and colocalization $\mathbf{v} 9$ algorithm $(p=0.0043)(n=3)$; e Representative transmission electron microscopy image (scale bar, $5 \mu \mathrm{m})(n=3)$; f Collagen quantification using Image J software $(p=0.0033)(n=9$ images, $N=3)$. $p$-value: unpaired two-tailed $t$-test performed in all comparisons. One-tail Fisher Exact test performed for KEGG pathway analysis. Data are represented as \pm SEM.

indicating that VDAC2 plays a crucial role in cardiac functioning by maintaining mitochondrial and cellular calcium homeostasis. Additionally, reintroduction of VDAC2 in young-KO mice showed a partial rescue of the cardiomyopathy phenotype suggesting the importance of VDAC2 in normal cardiac functioning.
Mitochondria-ER/SR interactions have long been studied and it is commonly believed that this interaction is crucial for mitochondrial calcium dynamics9,19,20. Mitochondria are known to recognize calcium-rich niches near SERCA2, RYR2, and NCX1 where major calcium transport happens ${ }^{21}$. We show in this study 

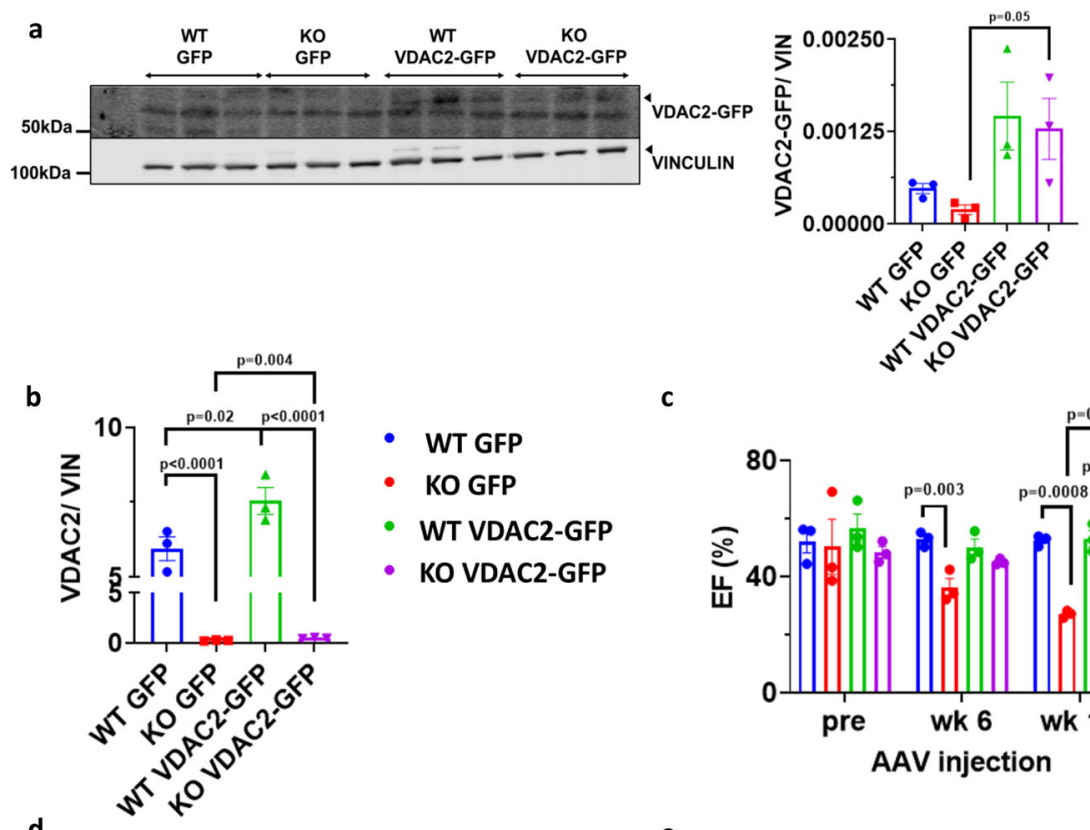

c
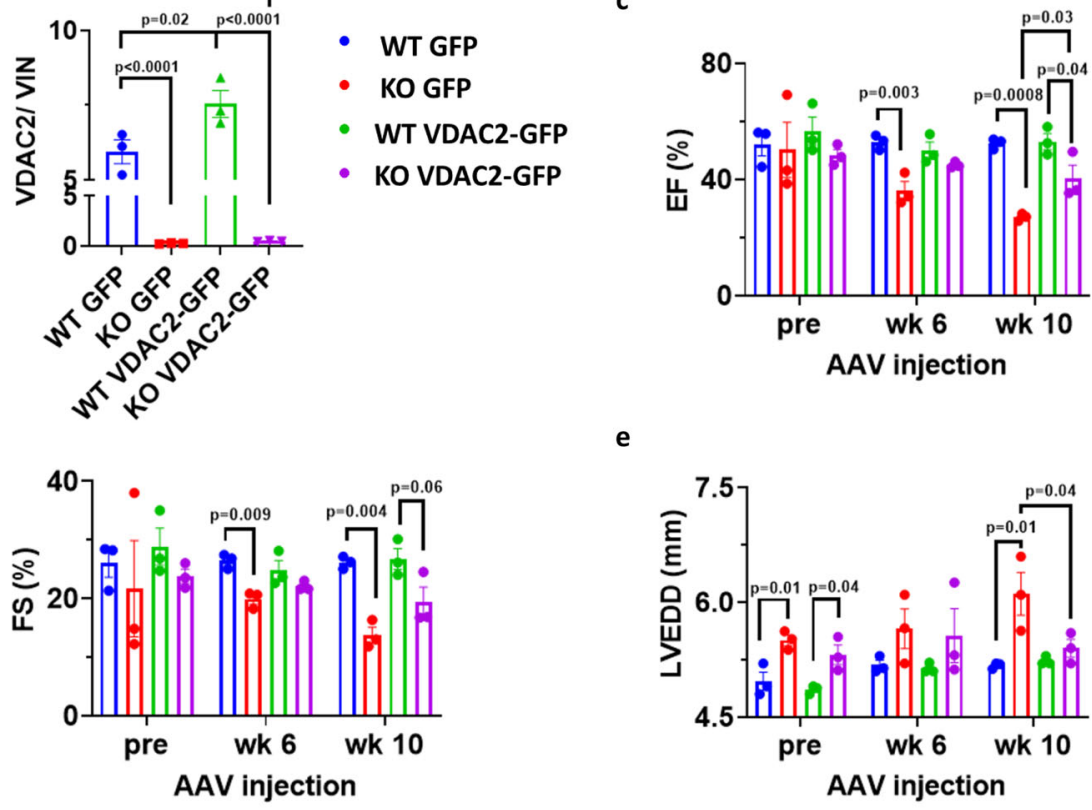

e

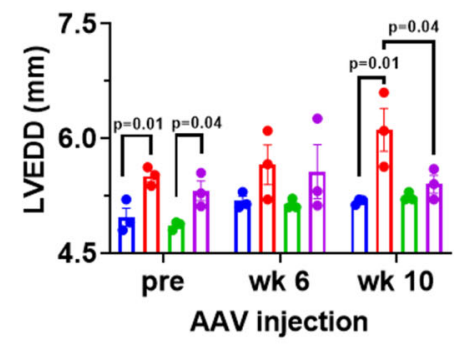

$\mathbf{f}$
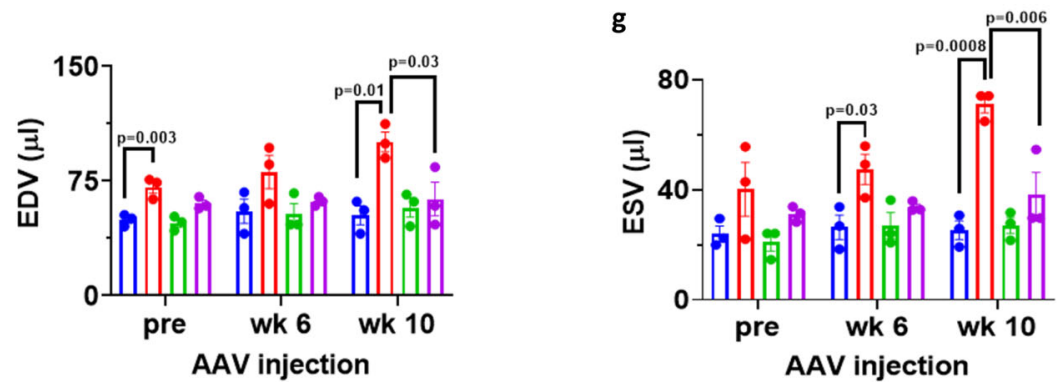

Fig. 8 Partial rescue of HF phenotype upon VDAC2 reintroduction. a Western blot image of VDAC2-GFP and lane-loading control VINCULIN and quantification using Image Studio Lite (version 5.2.5) (KO GFP vs. KO VDAC2-GFP $p=0.0568)(n=3)$; b qRT-PCR of Vdac2 normalized to Vcl (Vinculin) (KO GFP vs. KO VDAC2-GFP $p=0.0044)(n=3)$; $\mathbf{c}-\mathbf{g}$ Serial echocardiography parameter including ejection fraction (EF) (week 6: WT GFP vs. KO GFP $p=0.0036$, week 10: WT GFP vs. KO GFP $p=0.0008$, WT VDAC2-GFP vs. KO VDAC2-GFP $p=0.0456$, KO GFP vs. KO VDAC2-GFP $p=0.0359$ ), fractional shortening (FS) (week 6: WT GFP vs. KO GFP $p=0.0091$; week 10: WT GFP vs. KO GFP $p=0.0043$, WT VDAC2-GFP vs. KO VDAC2-GFP $p=$ 0.0652), left ventricular end diastolic diameter (LVEDD) (pre: WT GFP vs. KO GFP $p=0.0169$, WT VDAC2-GFP vs. KO VDAC2-GFP $p=0.0407$; week 10: WT GFP vs. KO GFP $p=0.0107$, KO GFP vs. KO VDAC2-GFP $p=0.0444$ ), end diastolic and end systolic volumes (EDV (pre: WT GFP vs. KO GFP $p=$ 0.0032; week 10: WT GFP vs. KO GFP $p=0.0104$, KO GFP vs. KO VDAC2-GFP $p=0.0378$ ) and ESV (week6: WT GFP vs. KO GFP $p=0.03$, week 10: WT GFP vs. KO GFP $p=0.0008$, KO GFP vs. KO VDAC2-GFP $p=0.0066)$ ) respectively $(n=3)$. $p$-value: unpaired $t$-test performed in all comparisons between KO GFP and KO VDAC2-GFP in panels $\mathbf{a}$ and $\mathbf{b}$. Ordinary one-way ANOVA was performed for echocardiographic data and panel $\mathbf{b}$. Data are represented as \pm SEM.

that the crosstalk of VDAC2 with both calcium release throughRYR2 and diastolic calcium removal by SERCA2 and NCX1 plays an important role in determining cellular calcium homeostasis and when this crosstalk is lost, there is a significant impairment in both mitochondrial and cytosolic calcium signaling. We also observed a significant reduction in most calcium handling proteins like RYR2, SERCA2, NCX1, and CASQ2 in VDAC2-KO mice. While these results are unexpected, they might be explained by different hypotheses: It is feasible that the lack of mitochondrial calcium uptake specifically through the loss of RYR2-VDAC2 connection results in a lack of energy demand which was shown to depend on mitochondrial calcium uptake ${ }^{22}$. In this scenario the basic metabolism of VDAC2-KO cells would be comparable to WT (as demonstrated in our results), while they are unable to adapt to higher workload under stress situations and might consecutively down-regulate calcium handling 

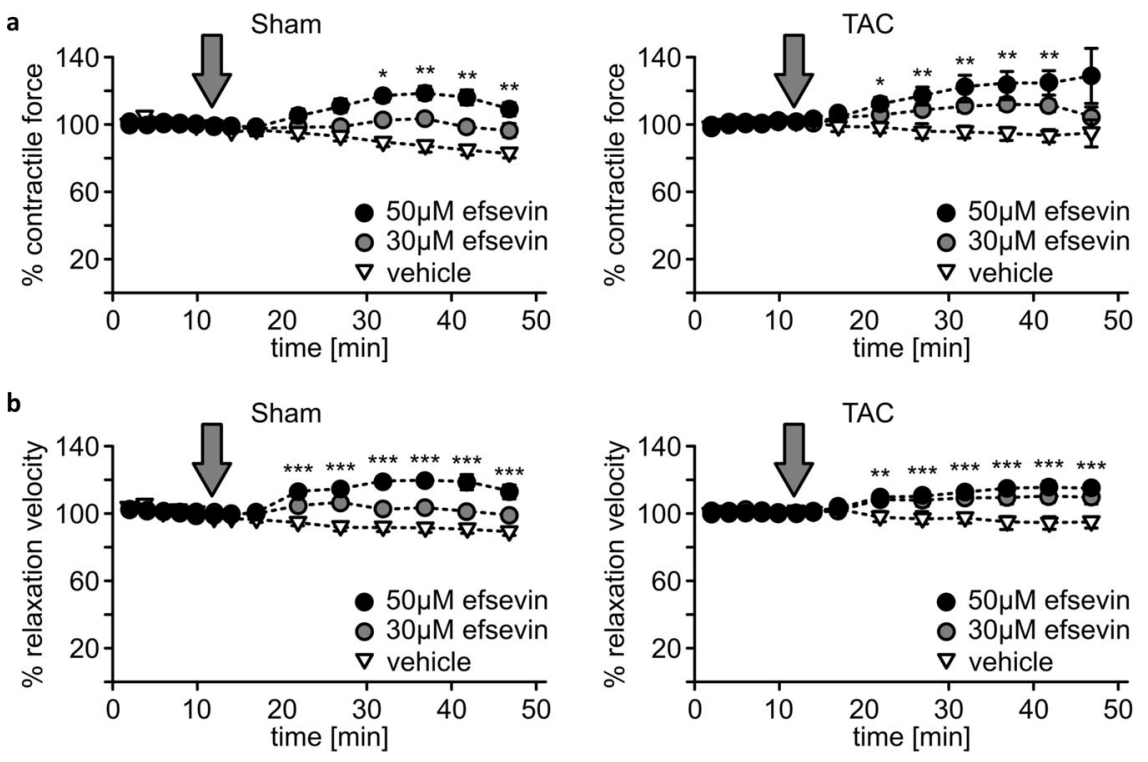

Fig. 9 Enhanced contractile force and accelerated relaxation speed in myocardial tissue upon VDAC2 activation. a Measurement of relative contractile force in organotypic myocardial slices from both Sham- (left panel) and TAC-operated mice (right panel) after superfusion with either 30 or $50 \mu \mathrm{M}$ of the VDAC2 agonist efsevin. Each dot represents the quantification of twitch amplitudes over $20 \mathrm{~s}$ at indicated time points. The gray arrow indicates the start of efsevin superfusion (left panel: DMSO vs. $30 \mu \mathrm{M}$ Efsevin: $10 \mathrm{~min} p=0.016,32 \mathrm{~min} p=0.010,37 \mathrm{~min} p=0.0004,42 \mathrm{~min} p=0.0012$ and $47 \mathrm{~min} p=$ 0.0062; DMSO vs. $50 \mu \mathrm{M}$ Efsevin: $22 \mathrm{~min} p=0.0007,27-47 \mathrm{~min} p<0.0001$, respectively) (right panel: DMSO vs. $30 \mu \mathrm{M}$ Efsevin: $17 \mathrm{~min} p=0.0038,22$ $\min p=0.0009,27 \min p=0.00031,32 \min p=0.00029,37 \min p=0.00015,42 \min p=0.00073 ;$ DMSO vs. $50 \mu \mathrm{M}$ Efsevin: $22 \mathrm{~min} p=0.0218,27$ $\min p=0.0044,32 \min p=0.0037,37 \min p=0.0031,42 \min p=0.0034)$. b Relaxation speed of twitches of myocardial slices shown in a $(N=8, n=$ 22 slices for TAC, $N=6, n=14$ slices for Sham) (left panel: DMSO vs. $30 \mu \mathrm{M}$ Efsevin: $22 \mathrm{~min} p=0.0072,27 \mathrm{~min} p=0.00019,32 \mathrm{~min} p=0.044,37 \mathrm{~min}$ $p=0.0125,42 \mathrm{~min} p=0.0145,47 \mathrm{~min} p=0.0308$; DMSO vs. $50 \mu \mathrm{M}$ Efsevin: $22-47 \mathrm{~min} p<0.0001$ respectively) (right panel: DMSO vs. $30 \mu \mathrm{M}$ Efsevin: $22 \min p<0.0001,27 \min p=0.0001,32 \min p<0.0001,37 \min p<0.0001,42 \min p=0.0001,47 \min p=0.0002 ;$ DMSO vs. $50 \mu \mathrm{M}$ Efsevin: 22 min $p=0.002,27 \mathrm{~min} p=0.0001,32-42 \mathrm{~min} p<0.0001$, respectively, $47 \mathrm{~min} p=0.0001)$. $p$-value: unpaired $t$-test performed in all comparisons. ${ }^{*}<0.05$, ${ }^{\star \star}<0.01$ and ${ }^{* \star \star}<0.001$. Data are represented as \pm SEM.

proteins. Alternatively, the down-regulation might be a result of compensatory mechanism to the slower rate of calcium uptake observed in the KO. Additionally, a down-regulation due to loss of structural integrity of the myocardium as observed in our experiment is feasible. Also, our data stands in contrast to previous observations that knock-down of VDAC2 in HL-1cells did not alter expression levels of calcium handling proteins ${ }^{10}$. However, major differences between the model systems might explain these discrepancies: Partial inhibition of VDAC2 ( 75\%) was achieved using viral transduction and cells were cultured in vitro for limited time. It is thus feasible that the down-regulation of these proteins is masked either by the remaining VDAC2 expression, the lack of triggers present in vivo-like pressure or adrenergic stimulation, or that these translational changes only manifest after a longer time.

The significant impairment in SR-mediated calcium release and uptake in VDAC2-KO mice can be associated with reduced RYR2 and SERCA2 activities, respectively. We also observed a significant downregulation of total and phosphorylated PLN in VDAC2-KO which may account for the reduced SERCA2 activity and slower rate of decline in calcium transient. Additionally, the varying molecular weights seen in total PLN protein is likely due to post-translational modification of PLN by phosphorylation at different sites or different combination of sites including Ser10, Ser16, and/or Thr17. Therefore, an average intensity of the bands was taken and normalized with GAPDH ${ }^{23-25}$. Our co-IP studies suggest an interaction of VDAC2 with SERCA2. We propose that this interaction might be important for facilitating mitochondrial calcium uptake and also indirectly influencing cytosolic calcium signaling. Another pathway for calcium extrusion from the cytoplasm is through NCX1 and we observed that VDAC2 interacts with NCX1. This interaction, similar to that with SERCA2 and RyR2, is also likely to influence cellular calcium signaling. Sub-cellular regions near RyR2, SERCA2, and NCX1 act as calcium compartments with markedly different calcium concentration than the bulk cytosol and both the interaction of VDAC2 with calcium handling proteins and the localization of mitochondria near these regions indicates a substantial role of mitochondrial calcium uptake for cellular calcium handling. We propose that upon disruption of this interaction, there is less overall mitochondrial calcium uptake and reduced cytosolic calcium clearance which leads to impaired intracellular calcium signaling (Supplementary Fig. 7).

In agreement with other studies, we showed that VDAC2 is crucial for cellular calcium cycling and normal cardiac functioning, thereby making it a promising therapeutic target for DCM and chronic HF ${ }^{6,15}$. To test this hypothesis, we investigated the potency of efsevin as a therapeutic agent for the treatment of HF. Indeed, efsevin enhanced contractile force in organotypic tissue slices of failing murine myocardium, establishing VDAC2 as a promising target for HF.

$\mathrm{HF}$ is associated with an increased risk for arrhythmia and efsevin was previously suggested to suppress arrhythmogenesis in cardiomyocytes $^{13,14}$ suggesting a role for VDAC2 also as a protective pathway against arrhythmia. This data is backed up by our findings, that VDAC2-KO mice also experienced arrhythmias. We hypothesize that the altered calcium signaling (reduced CASQ2, RYR2, and SERCA2 activities) has the potential to cause these arrhythmias ${ }^{26,27}$. The reduced CASQ2 activity suggests reduced RyR2 sensitivity to cytosolic calcium which in turn explains the reduced gene expression of L-type calcium channel ${ }^{28}$. Additionally, the expression of T-type calcium channels in the 
adult $\mathrm{KO}$ myocardium is indicative of disease states such as cardiomyopathies and HF where increased expression of T-type channels in adult ventricular myocytes leads to increased susceptibility to arrhythmias ${ }^{29,30}$. The cellular patch-clamp experiments in the VDAC2-KO showed a tendency towards increased early after depolarizations perhaps due to reactivation of these calcium channels ${ }^{31}$. We also observed a significantly longer $\mathrm{APD}_{50}$ in the $\mathrm{KO}$ which can be explained by the genetic downregulation of the transient outward channel thereby making VDAC2 influence on the cardiomyocyte even more prominent.

VDAC2-tubulin interaction and VDAC2-PFKP interaction have been studied in cancer metabolism research were specifically targeting VDAC2 has shown to down-regulate glycolysis thereby preventing cancer cell growth and proliferation ${ }^{32,33}$. In agreement with this work, we also observed significant alterations in a number of enzymes involved in glucose metabolism, its accessory pathways, and the TCA cycle but no difference was observed in FAO or electron transport chain (ETC). This unaltered FAO and ETC may help explain the relatively unaltered mitochondrial respiration since these metabolic pathways provide the necessary ATP.

VDAC2 plays an important role in apoptosis by interacting with BAK, a proapoptotic protein, and keeping it inactive ${ }^{34}$. Essentially, VDAC2-BAK interaction prevents cellular apoptosis, and $V$ dac2 deletion results in free (or active) BAK thus causing excessive apoptosis ${ }^{35}$. We hypothesize that this increased apoptosis during late cardiac development might contribute to the increased fibrosis observed in our VDAC2-KO mice.

Calcium release into the cytoplasm is mainly mediated by the RyR2 and previous publications have established the importance of physical interaction between VDAC2 and RyR2 for normal calcium transport into the mitochondria ${ }^{10}$. Mitochondrial calcium uptake was significantly impaired in our VDAC2-KO mice indicating the importance of VDAC2 in mitochondrial calcium transport. A similar reduction in mitochondrial calcium uptake was observed in MCU-KO mice, however, no other phenotypical difference was observed between the MCU-WT and MCU-KO ${ }^{36}$, indicating differences in the role of these two transporters incellular calcium handling. One explanation derived from this work is that VDAC might act as a scaffolding protein to link the MCU to other calcium handling proteins and thus creating cellular calcium microdomains, while MCU might serve as the controller of mitochondrial calcium uptake. This is in line with observations that VDAC2 was described to be a vividly interacting channel ${ }^{20}$.

Overall, our findings highlight the importance of VDAC2 in influencing ECC and cardiac disease progression. Previous research has shown that increased calcium uptake through VDAC2 (using efsevin) reverses arrhythmic phenotypes. Here, we demonstrate the crucial role of VDAC2 in ECC and the pathogenesis of DCM and provide reasonable evidence for VDAC2 to serve as a therapeutic target for HF. Thus, drugs targeting VDAC2 might fulfill a double role by suppressing cardiac arrhythmia and enhancing cardiac function.

\footnotetext{
Methods

Animals and animal care. All animal studies were performed in accordance with the University of Iowa Animal Care and Use Committee (IACUC). All procedures involving animals were approved by the Animal Care and Use Committee of the University of Utah and complied with the American Physiological Society's Guiding Principles in the Care and Use of Animals and the UK Animals (Scientific Procedures) Act 1986 guidelines. The mice were housed in $12 \mathrm{~h}$ dark/light cycle at $70{ }^{\circ} \mathrm{F}$ and $40 \%$ humidity.
}

Generation of cardiac-specific knockout. C57BL6J mice were used for all experiments. Both male and female mice were used for all experiments. Vdac2 flox/ flox mice were obtained from Ren et al. ${ }^{35}$. The founder mice were cross-bred with transgenic aMHC-Cre (Jackson Laboratory\#009074) ${ }^{37}$ and the resulting heterozygous (Vdac2 flox/+; aMHC-Cre) mice were crossed to obtain a cardiac ventricular myocyte-specific deletion of VDAC2.

Echocardiographic analysis. Mice were anesthetized with $1.5 \%$ Isoflurane (Vet One, NDC13985-046-60) during echocardiography. Echocardiographic images were taken on the Vivo system. Echoes were performed serially from embryonic age 17 to 16 weeks post-natal. 2D long-axis and short-axis views were obtained and used for analysis using Vivo strain software (version 3.1.1). Two consecutive cardiac cycles were used for all the measurements. Limb leads were used to record an electrocardiogram (ECG).

Collagen content evaluation/Masson's trichrome stain. Mice were euthanized using sodium pentobarbital (NDC76478-501-50). Hearts were harvested and formalin-fixed for $48 \mathrm{~h}$ followed by paraffin embedding. $5 \mu \mathrm{m}$ thick sections were cut and stained with Trichrome for fibrosis analysis using the Dako automated special strainer. The slides for fibrosis analysis were scanned under $\times 20$ and analyzed using Aperio Image Scope software (version 12.3.2.8013) (using the colocalization v9 algorithm $)^{38}$. A ratio of the total stained area to the collagen-stained area was reported.

Cardiomyocyte isolation. Adult mice were anesthetized with sodium pentobarbital $\left(50 \mathrm{mg} \mathrm{kg}^{-1}\right)$ and the excised heart was attached to an aortic cannula and perfused with solutions gassed with $100 \% \mathrm{O}_{2}$ and held at $37^{\circ} \mathrm{C}, \mathrm{pH}$ 7.3. Perfusion with a $0 \mathrm{mM} \mathrm{Ca}^{2+}$ solution for $5 \mathrm{~min}$ was followed by $15 \mathrm{~min}$ of perfusion with the same solution containing $1 \mathrm{mg} \mathrm{ml}^{-1}$ collagenase (type II, Worthington Biochemical, Freehold, NJ, USA) and $0.1 \mathrm{mg} / \mathrm{ml}$ protease (type XIV, Sigma Chemical, catalog \#P5147). The heart was then perfused for 1 min with a stopping solution (the same solution containing $20 \%$ serum and $0.2 \mathrm{mM} \mathrm{CaCl}_{2}$ ). All perfusions were performed at a flow rate of $2 \mathrm{ml} \mathrm{min}^{-1}$. The atria were removed and the ventricles were minced and shaken for $10 \mathrm{~min}$, and then filtered through a nylon mesh. Cells were stored at $37^{\circ} \mathrm{C}$ in a normal HEPES buffered solution. All myocytes used in this study were rod-shaped, had well-defined striations, and did not spontaneously contract. Experiments were performed within $7 \mathrm{~h}$ of isolation.

Cell super-fusion chamber. The Plexiglas cell bath had a clear glass bottom and was mounted on the stage of an inverted microscope (Diaphot, Nikon, Japan). The temperature of the solutions in the super-fusion chamber was $36 \pm 0.3^{\circ} \mathrm{C}$. Bathing solutions flowed continuously through the bath at $4 \mathrm{ml} \mathrm{min}^{-1}$, and solution depth was held at $\sim 2-3 \mathrm{~mm}$. Exchange of the bath solution required $\sim 5 \mathrm{~s}$. The bottom of the bath was coated with laminin (Collaborative Research, Bedford, MA, USA) to improve cell adhesion.

Cell bathing solutions and pipette filling solutions. The normal control bathing solution contained (mM): $126.0 \mathrm{NaCl}, 11.0$ dextrose, $4.4 \mathrm{KCl}, 1.0 \mathrm{MgCl}_{2}, 1.08$ $\mathrm{CaCl}_{2}$, and 24.0 HEPES titrated to $\mathrm{pH} 7.4$ with $1 \mathrm{M} \mathrm{NaOH}$. The pipette solution used for recording APs contained (mM): $110.0 \mathrm{KCl}, 5.0 \mathrm{NaCl}, 5.0 \mathrm{MgATP}, 5.0$ phosphocreatine, 1.0 NaGTP, 10.0 HEPES titrated to $\mathrm{pH} 7.2$ with $1 \mathrm{M} \mathrm{KOH}$.

AP measurement. Transmembrane potential $\left(V_{\mathrm{m}}\right)$ was measured with borosilicate glass suction pipettes (resistance 1-2 $\mathrm{M} \Omega$ when filled) connected to an Axoclamp $2 \mathrm{~B}$ amplifier system (Axon Instruments/Molecular Devices) in bridge mode ${ }^{39}$. The $V_{\mathrm{m}}$ signal was filtered at $5 \mathrm{kHz}$, digitized at $50 \mathrm{kHz}$ with a 16 -bit A/D converter (Digidata 1322A), and analyzed using PCLAMP 8 software (Molecular Devices). APs were triggered with brief $(2-3 \mathrm{~ms})$ square pulses of depolarizing intracellular current $(\sim 2 \mathrm{nA})$. $\mathrm{APD}_{50}$ and $\mathrm{APD}_{90}$ were measured.

Intracellular calcium concentration measurement. Calcium transients were detected in single myocytes with an epifluorescence system using the fluorescent indicator, Fluo-4. Cells were incubated in the normal control solution containing $12.5 \mu \mathrm{M}$ Fluo4-AM (Invitrogen, catalog\#F14201) and $0.3 \mathrm{mM}$ probenecid (Sigma, catalog \#P8761) at $30^{\circ} \mathrm{C}$ for $20 \mathrm{~min}$. The cells were then continuously bathed in the same solution containing no indicator. Probenecid $(0.3 \mathrm{mM})$ was included in the bathing solutions to help retard fluo- 4 loss from the cells. Fluorescence emission ( $535 \mathrm{~nm}$, bandpass filter) was collected with a photomultiplier tube via a $\times 40$ objective during continuous excitation at $485 \mathrm{~nm}$. Calcium transients were elicited with field stimulation at a cycle length of $1 \mathrm{~s}$.

TEM and analysis. LV transmural samples from 16-week-old mice were used for this study. Tissue samples were fixed overnight at $4{ }^{\circ} \mathrm{C}$ in $0.1 \mathrm{M}$ sodium cacodylate buffer containing $1 \%$ paraformaldehyde and $2.5 \%$ glutaraldehyde. Samples were washed with the same buffer and post-fixed for $2 \mathrm{~h}$ in $2 \%$ osmium tetroxide buffered with cacodylate buffer. Samples were then rinsed in nano-pure water and stained with en-bloc stain for $1 \mathrm{~h}$ at room temperature in uranyl acetate. Tissues were then dehydrated through a graded series of ethanol: $10 \mathrm{~min}$ in $50 \%, 3 \times$ $10 \mathrm{~min}$ in $70 \%, 2 \times 10 \mathrm{~min}$ in $95 \%, 4 \times 10 \mathrm{~min}$ in absolute ethanol, and $3 \times 10 \mathrm{~min}$ with absolute acetone. Samples were incubated at room temperature in a gradually 
Table 1 List of antibodies used.

Voltage-dependent anion-selective channel protein 2 (VDAC2)

Voltage-dependent anion-selective channel protein 1 (VDAC1)

Voltage-dependent anion-selective channel protein 3 (VDAC3)

Vinculin

Glyceraldehyde 3-phosphate dehydrogenase (GAPDH)

Transketolase (TKT)

Methylenetetrahydrofolate dehydrogenase 1 (MTHFD1)

Aldolase B (ALDOB)

Serine hydroxymethyltransferase 1 (SHMT1)

Isocitrate dehydrogenase (IDH1/2)

Phosphofructokinase 1 (PFK1)

Pyruvate carboxylase (PC)

Pyruvate dehydrogenase (PDH)

Lactate dehydrogenase (LDH)

Hexokinase 1 (HK1)

Calsequestrin 2 (CASQ2)

Citrate synthase (CS)

Succinyl Co-A synthetase (SUCLG2)

Succinate dehydrogenase A (SDHA)

Sodium-calcium exchanger 1 (NCX1)

Sarcoplasmic endoplasmic reticular calcium ATPase 2 (SERCA2)

Ryanodine receptor 2 (RYR2)

Phospholamban (PLN)

Phospho-phospholamban (pPLN)

Protein kinase A (PKA)

Phospho-protein kinase A (pPKA)

Mitochondrial calcium uniporter (MCU)

Mitochondrial sodium calcium exchanger (NCLX)

Dynamin-related protein 1 (DRP1)

Oxidative phosphorylation (OXPHOS)

IRDye 680RD Donkey anti-Mouse Secondary

IRDye 800CW Donkey anti-Rabbit Secondary

Co-immunoprecipitation-antibodies

Voltage-dependent anion-selective channel protein 2 (VDAC2)

Immunoglobulin G (IgG)

Immunofluorescence/proximity ligation assay-antibodies/stain

4',6-diamidino-2-phenylindole (DAPI)

Voltage-dependent anion-selective channel protein 2 (VDAC2)

Sodium-calcium exchanger 1 (NCX1)

Sarcoplasmic endoplasmic reticular calcium ATPase 2 (SERCA2)

Ryanodine receptor 2 (RYR2)

Quantitative reverse transcription-PCR-primers

Voltage-dependent anion-selective channel protein 2 (VDAC2)

Vinculin

\begin{tabular}{|c|c|}
\hline 1:1000 & $9412 \mathrm{~S}$ \\
\hline 1:1000 & ab14734 \\
\hline 1:1000 & ab130561 \\
\hline 1:1000 & $13901 \mathrm{~s}$ \\
\hline 1:1000 & $5174 S$ \\
\hline $1: 500$ & $8616 S$ \\
\hline $1: 500$ & sc-271412 \\
\hline $1: 200$ & sc-365449 \\
\hline $1: 500$ & 80715 \\
\hline 1:500 & sc-373816 \\
\hline $1: 500$ & sc-166722 \\
\hline 1:1000 & NBP1-49536 \\
\hline $1: 500$ & $3205 S$ \\
\hline 1:1000 & $3582 S$ \\
\hline $1: 500$ & $2024 S$ \\
\hline $1: 500$ & ab3516 \\
\hline 1:1000 & 143095 \\
\hline 1:1000 & 80715 \\
\hline $1: 1000$ & $5839 S$ \\
\hline $1: 500$ & ab151608 \\
\hline $1: 500$ & $4388 S$ \\
\hline $1: 500$ & MA3-916 \\
\hline 1:1000 & $14562 S$ \\
\hline 1:1000 & 84965 \\
\hline $1: 500$ & SAB4502337 \\
\hline $1: 500$ & SAB4503969 \\
\hline $1: 500$ & 14997S \\
\hline $1: 500$ & SAB2102181 \\
\hline $1: 500$ & $14647 \mathrm{~S}$ \\
\hline 1:1000 & ab110413 \\
\hline $1: 10,000$ & $926-68072$ \\
\hline $1: 10,000$ & $926-32212$ \\
\hline $5 \mu g$ & PA5-28106 \\
\hline $5 \mu g$ & ACO05 \\
\hline 1:1000 & D3571 \\
\hline 1:1000 & 66388-1-IG \\
\hline 1:500 & ab151608 \\
\hline 1:500 & $4388 S$ \\
\hline 1:500 & MA3-916 \\
\hline
\end{tabular}

94125

ab13056

139015

sc-271412

sc-365449

sc-166722

NBP1-49536

35825

2024S

ab3516

14309

58395

ab151608

4388S

$14562 \mathrm{~S}$

84965

SAB4502337

AB4503969

SAB2102181

146475

ab110413

926-68072

PA5-28106

D3571

66388-1-IG

MA3-916

Mm00834279_m1 Mm00447745_m1
Cell Signaling Technology

Abcam

Abcam

Cell Signaling Technology

Cell Signaling Technology

Cell Signaling Technology

Santa Cruz Biotech

Santa Cruz Biotech

Cell Signaling Technology

Santa Cruz Biotech

Santa Cruz Biotech

Novus Bio

Cell Signaling Technology

Cell Signaling Technology

Cell Signaling Technology

Abcam

Cell Signaling Technology

Cell Signaling Technology

Cell Signaling Technology

Abcam

Cell Signaling Technology

Thermofisher Scientific

Cell Signaling Technology

Cell Signaling Technology

Sigma Aldrich

Sigma Aldrich

Cell Signaling Technology

Sigma Aldrich

Cell Signaling Technology

Abcam

LI-COR Biosciences

LI-COR Biosciences

Thermofisher Scientific AbClonal

Thermofisher Scientific Thermofisher Scientific Abcam

Cell Signaling Technology

Thermofisher Scientific

Thermofisher Scientific Thermofisher Scientific increasing concentration of epoxy resin (Electron Microscopy Science, Hatfield, PA) to facilitate infiltration. Samples were then transferred to $50 \%$ resin in acetone for $1 \mathrm{~h}$ and overnight in $75 \%$ resin in acetone. Samples were then transferred to $100 \%$ resin for $8 \mathrm{~h}$ with three fresh resin changes. Samples were then embedded and polymerized at $60^{\circ} \mathrm{C}$ for $48 \mathrm{~h}$. Ultrathin sections $(70 \mathrm{~nm})$ were obtained with a diamond knife (Diatome) using Leica UC6 (Leica Microsystems, Vienna, Austria). Sections were post-stained with saturated uranyl acetate for $10 \mathrm{~min}$ followed by Reinold stain for $5 \mathrm{~min}$. Sections were imaged at $120 \mathrm{kV}$ with JEOL $1400 \mathrm{Plus}^{40}$. Mitochondrial density and number analysis were performed using Adobe Photoshop tool using $\times 1500$ images. A $22 \times 14$ grid was used for density measurements. The same images were used for collagen quantification using Fiji software. The ratio of collagen area to total area was reported.

Mitochondria isolation and calcium uptake. Mice were euthanized using carbondioxide for $3 \mathrm{~min}$ and the heart was excised. The atria were removed and the ventricles were used for mitochondria isolation using differential centrifugation ${ }^{41}$. Protein estimation was performed using Pierce Assay. $100 \mu \mathrm{g}$ of protein was used per trial, resuspended in $100 \mu \mathrm{L}$ of a solution containing (in mM): $125.0 \mathrm{KCl}, 20.0$ HEPES, 10.0 K $\mathrm{HPO}_{4}$, 5.0 Glutamate, 5.0 Malate, and $1 \mu \mathrm{M}$ Oregon Green BAPTA/6F ( $\mathrm{pH} 7.2$ with $1 \mathrm{M} \mathrm{KOH}$ ). Imaging was performed on a Biotek Cytation 5 instrument in 96-well flat-bottomed plates. Baseline measurements were taken for $30 \mathrm{~s}$ and $12 \mu \mathrm{M}$ calcium chloride was injected and the fluorescence (excitation
$490 \mathrm{~nm}$, emission $517 \mathrm{~nm}$ ) was measured for 3 min to obtain the calcium uptake slope.

Retro-orbital injections. 6-week-old WT and KO mice were randomly chosen to receive either AAV9-aMHC-VDAC2-GFP vector or control AAV9-aMHC-GFP vector. $N=3$ mice were used in each group and a total of four groups were used (WT-AAV and KO-AAV received the vector with VDAC2 and WT-GFP and KOGFP received control vector). A concentration of $3.1 \times 10 \mathrm{e} 12 \mathrm{VG} \mathrm{kg}^{-1}$ was used for each injection and the mice were serially echoed for 10 weeks post-injection.

RNA extraction, sequencing, and qRT-PCR. miRNeasy Mini kit (Qiagen) was used for RNA extraction from the LV transmural sample. The extracted RNA was used for total RNA sequencing (RNA Seq). Agilent RNA Screen Tape Assay was used for QC experiments. Illumina TruSeq Stranded RNA kit was used for library preparation and Ribo-Zero Gold was used to remove rRNA and the sequencing was performed on an Illumina HiSeq 2500 with $50 \mathrm{bp}$ single-end reads. The same RNA was used for cDNA synthesis (NEB \#E3010S) and qRT-PCR was performed using Vdac2 and $\mathrm{Vcl}$ (VINCULIN) primers (Table 1).

RNA Seq analysis. RNA Seq analysis was performed with the High-Throughput Genomics and Bioinformatics Analysis Shared Resource at Huntsman Cancer 
Institute at the University of Utah. mm10, M_musculus_Dec_2011, GRCm38 genome build was used for sequence alignment. Sample outliers were checked by summarizing the output files from cutadapt, FastQC, Picard CollectRnaSeqMetrics, STAR, and feature counts by using the previously described method ${ }^{42} .5 \%$ false discovery rate (FDR) with DeSeq2 (version 1.24.0) was used to identify differentially expressed genes ${ }^{43}$. Genes were filtered using the following criteria: adjusted $p$ value $<0.05$, absolute $\log 2$ fold change $>0.585$ and normalized base mean $>30^{43}$. Heat maps were produced using the R library $p$ heatmap. KEGG ${ }^{44}$ and Ingenuity pathway analysis (IPA) software was used for gene ontology analysis.

Protein extraction and Western blotting. WT and KO mice hearts ( $n=6$ each) were excised. $30 \mu \mathrm{g}$ of transmural LV sample was homogenized using metal beads for $3 \mathrm{~min}$ in RIPA buffer with $2 \times$ protease and phosphatase inhibitor (ThermoScientific \#1861281). The homogenate was transferred to a new tube containing $10 \mu \mathrm{l}$ of $1 \times \mathrm{PMSF}$ and allowed to rotate for $30 \mathrm{~min}$ at $4^{\circ} \mathrm{C}$ and centrifuged at $11,000 \times g$ for $10 \mathrm{~min}$ at $4^{\circ} \mathrm{C}$. The supernatant was used for protein estimation using the Pierce BCA Protein Assay kit (Thermo-Scientific \#23225). An equal volume of $2 \times$ Laemmli buffer (with 10\% DTT) was added to the sample and boiled for $10 \mathrm{~min}$ at $98^{\circ} \mathrm{C}$

$30 \mu \mathrm{g}$ protein was used for SDS-PAGE. The gel was run at constant volts $(50 \mathrm{~V})$ and proteins were transferred to nitrocellulose membrane at constant current $(350 \mathrm{~mA})$. For RYR2, proteins were transferred at constant volt $(40 \mathrm{~V})$ overnight. The membranes were blocked for $1 \mathrm{~h}$ with $5 \%$ non-fat milk and probed with primary antibodies overnight in the cold room. Antibodies and the concentration used are listed in Table 1. Blots were washed with $1 \times$ PBST thrice and probed with a secondary antibody for $1 \mathrm{~h}$. Blots were washed with $1 \times$ PBST thrice prior to scanning using LI-COR. Image Studio Lite software was used to analyze the protein blots. Each blot had its own lane loading control and the values were normalized accordingly.

Co-immunoprecipitation. $30 \mu \mathrm{g}$ of LV transmural sample from 8-week-old WT and $\mathrm{KO}$ mice were used for protein extraction and quantification was done using a BCA assay kit. Protein A magnetic beads (Bio-Rad, catalog\#161-4011) were washed thrice with $1 \times$ PBST. $4 \mathrm{mg} \mathrm{ml}^{-1}$ of lysate was precleared by rotating with beads for $30 \mathrm{~min}$ at $4{ }^{\circ} \mathrm{C}$ and $10 \%$ of the precleared lysate was saved as input. $5 \mu \mathrm{g}$ of antibody (Table 1) was used for conjugation with the beads by rotating at room temperature for $10 \mathrm{~min}$, followed by washing twice with $1 \times$ PBST and once with the lysis buffer (Cell Signaling, catalog \#9806). The precleared lysate and antibody were kept for rotation at room temperature for $1 \mathrm{~h}$. The magnet was then washed thrice with $1 \times$ PBST, magnetized and the elute was boiled at $70{ }^{\circ} \mathrm{C}$ for $10 \mathrm{~min}$ with $40 \mu \mathrm{l}$ of $1 \times$ Laemmli buffer ( $10 \% \beta$-mercaptoethanol) and used for western blot.

Immunofluorescence. 16-week-old mice hearts were fixed in 10\% paraformaldehyde for $4-6 \mathrm{~h}$ followed by paraffin embedding. Samples were cut at $5 \mu \mathrm{m}$ thickness and deparaffinized and antigen retrieval was performed. The antibodies used are listed in Table 1. Secondary anti-mouse, anti-rabbit, or anti-goat were used appropriately. All images for each protein were acquired using the same laser setting and processed using Fiji software.

Proximity ligation assay. 16-week-old mice heart samples embedded in paraffin were used for this assay. Duolink In Situ PLA probe anti-mouse, anti-rabbit, and anti-goat were used (DUO92001, DUO92005, and DUO92015). The antibodies used are listed in Table 1. Samples were processed using the manufacturer's protocol. All images for each combination were acquired using the same laser settings and processed using Fiji software.

Force measurements in murine organotypic slices. Hearts from adult mice were excised after cervical dislocation and flushed in ice-cold preparation buffer (in $\mathrm{mM}$ ): $136.0 \mathrm{NaCl}, 5.4 \mathrm{KCl}, 1.0 \mathrm{MgHPO}_{4}, 0.9 \mathrm{CaCl}_{2}, 30.0$ 2,3-Butanedionemonoxim, 5.0 HEPES, pH 7.4. For sectioning, hearts were embedded in low melting agarose and cut into $300 \mu \mathrm{m}$-thick coronary plane slices using a LeicaVT1200S vibratome and glued (Histoacryl, B. Brain Melsungen AG, Germany) onto small plastic triangles cut from $0.1 \mathrm{~mm}$-thick polyester copier clear before being mounted in an organ bath chamber (Mayflower, Hugo Sachs Elektronic; HSE, Germany). Contractility was measured under a constant flow-through $\left(4 \mathrm{ml} \mathrm{min}^{-1}\right)$ in prewarmed measuring buffer (in $\mathrm{mM}$ ): $136.0 \mathrm{NaCl}, 5.4 \mathrm{KCl}, 1.0 \mathrm{MgCl}_{2}, 0.33$ $\mathrm{NaH}_{2} \mathrm{PO}_{4}, 10.0$ Glucose, $1.8 \mathrm{CaCl}_{2}, 23.0 \mathrm{NaHCO}_{3}, \mathrm{pH} 7.4,37^{\circ} \mathrm{C}$ under electrical pacing at $3 \mathrm{~Hz}$ and force was recorded by a force transducer connected to an amplifier (Hugo Sachs, Germany) using WinEDR (University of Strathclyde, UK). Kinetics of single twitches was analyzed using LabChart Reader (ADInstruments).

TAC surgery. TAC ${ }^{45}$ was performed on RyR2 ${ }^{\mathrm{R} 4496 \mathrm{C}+/-}$ knock-in mice ${ }^{46}$, which were previously described to develop a severe form of $\mathrm{HF}^{47}$. In brief, 8-14-weekold mice were anesthetized by medetomidine/midazolam/fentanyl $(0.5,5.0$, and $0.05 \mathrm{mg} \mathrm{kg}^{-1}$ body weight intraperitoneally, respectively) and the aorta was constricted around a 27 -gauge needle. Sham mice underwent the same procedure without aortic constriction. 6 weeks after surgery, mice were sacrificed by cervical dislocation, hearts were isolated and flushed (in $\mathrm{mM}$ ): $136.0 \mathrm{NaCl}, 5.4 \mathrm{KCl}, 1.0$
$\mathrm{MgCl}_{2}, 0.33 \mathrm{NaH}_{2} \mathrm{PO}_{4}, 10.0$ Glucose, $0.9 \mathrm{CaCl}_{2}, 30.0$ 2,3-butanedionemonooxime, 5.0 HEPES, $\mathrm{pH} 7.4,4{ }^{\circ} \mathrm{C}$ and success of TAC surgery was confirmed by measuring the heart weight to tibia length ratio.

Statistics and reproducibility. All data were summarized as \pm SEM. GraphPad Prism (version8.2.1) was used for all statistical analyses. A two-tailed test was performed in all comparisons. Unpaired Student's $t$-test was used for all analyses with WT and KO. Multiple $t$-test and one-way ANOVA were used for longitudinal echocardiographic data and while comparing multiple groups, respectively. $p$-value $<0.05$ was considered statistically significant. All experiments were repeated independently (at least three biological replicates, and three technical replicates wherever applicable) with reproducible results. Samples used for western blot quantification were from the same gel and when unavoidable, were processed in parallel.

Reporting summary. Further information on research design is available in the Nature Research Reporting Summary linked to this article.

\section{Data availability}

Source data are provided within this manuscript as a source data file. RNA sequencing is uploaded to NCBI's GEO database under accession code GSE168487. Source data are provided with this paper.

Received: 28 May 2020; Accepted: 6 July 2021; Published online: 28 July 2021

\section{References}

1. Luscher, T. F. Heart failure: the cardiovascular epidemic of the 21 st century. Eur. Heart J. 36, 395-397 (2015).

2. van der Meer, P., Gaggin, H. K. \& Dec, G. W. ACC/AHA versus ESC guidelines on heart failure: JACC guideline comparison. J. Am. Coll. Cardiol. 73, 2756-2768 (2019).

3. Hasenfuss, G. et al. Alteration of contractile function and excitation-contraction coupling in dilated cardiomyopathy. Circ. Res. 70, 1225-1232 (1992)

4. Bers, D. M. Cardiac excitation-contraction coupling. Nature 415, 198-205 (2002).

5. Eisner, D. A., Caldwell, J. L., Kistamas, K. \& Trafford, A. W. Calcium and excitation-contraction coupling in the heart. Circ. Res. 121, 181-195 (2017).

6. Naghdi, S. \& Hajnoczky, G. VDAC2-specific cellular functions and the underlying structure. Biochim. Biophys. Acta 1863, 2503-2514 (2016).

7. Cheng, E. H., Sheiko, T. V., Fisher, J. K., Craigen, W. J. \& Korsmeyer, S. J. VDAC2 inhibits BAK activation and mitochondrial apoptosis. Science 301, 513-517 (2003)

8. Roy, S. S., Ehrlich, A. M., Craigen, W. J. \& Hajnoczky, G. VDAC2 is required for truncated BID-induced mitochondrial apoptosis by recruiting BAK to the mitochondria. EMBO Rep. 10, 1341-1347 (2009).

9. Eisner, V., Csordas, G. \& Hajnoczky, G. Interactions between sarcoendoplasmic reticulum and mitochondria in cardiac and skeletal musclepivotal roles in $\mathrm{Ca}(2)(+)$ and reactive oxygen species signaling. J. Cell Sci. 126, 2965-2978 (2013).

10. Min, C. K. et al. Coupling of ryanodine receptor 2 and voltage-dependent anion channel 2 is essential for $\mathrm{Ca}(2)+$ transfer from the sarcoplasmic reticulum to the mitochondria in the heart. Biochem. J. 447, 371-379 (2012)

11. Pan, $\mathrm{X}$. et al. The physiological role of mitochondrial calcium revealed by mice lacking the mitochondrial calcium uniporter. Nat. Cell Biol. 15, 1464-1472 (2013).

12. Murphy, E. et al. Unresolved questions from the analysis of mice lacking MCU expression. Biochem. Biophys. Res. Commun. 449, 384-385 (2014).

13. Shimizu, H. et al. Mitochondrial $\mathrm{Ca}(2+)$ uptake by the voltage-dependent anion channel 2 regulates cardiac rhythmicity. Elife 4, 04801 (2015)

14. Schweitzer, M. K. et al. Suppression of arrhythmia by enhancing mitochondrial $\mathrm{Ca}(2+)$ uptake in catecholaminergic ventricular tachycardia models. JACC Basic Transl. Sci. 2, 737-747 (2017)

15. Subedi, K. P. et al. Voltage-dependent anion channel 2 modulates resting Ca $(2)+$ sparks, but not action potential-induced $\mathrm{Ca}(2)+$ signaling in cardiac myocytes. Cell Calcium 49, 136-143 (2011)

16. Navankasattusas, $\mathrm{S}$. et al. The netrin receptor UNC5B promotes angiogenesis in specific vascular beds. Development 135, 659-667 (2008).

17. Landstrom, A. P., Dobrev, D. \& Wehrens, X. H. T. Calcium signaling and cardiac arrhythmias. Circ. Res. 120, 1969-1993 (2017).

18. Kwong, J. Q. et al. The mitochondrial calcium uniporter selectively matches metabolic output to acute contractile stress in the heart. Cell Rep. 12, 15-22 (2015). 
19. Sharma, V. K., Ramesh, V., Franzini-Armstrong, C. \& Sheu, S. S. Transport of $\mathrm{Ca} 2+$ from sarcoplasmic reticulum to mitochondria in rat ventricular myocytes. J. Bioenerg. Biomembr. 32, 97-104 (2000).

20. Sander, P., Gudermann, T. \& Schredelseker, J. A Calcium guard in the outer membrane: is VDAC a regulated gatekeeper of mitochondrial calcium uptake? Int. J. Mol. Sci. 22, 22020946 (2021).

21. Dewenter, M., von der Lieth, A., Katus, H. A. \& Backs, J. Calcium signaling and transcriptional regulation in cardiomyocytes. Circ. Res. 121, 1000-1020 (2017).

22. Morkin, E. Control of cardiac myosin heavy chain gene expression. Microsc. Res. Tech. 50, 522-531 (2000).

23. Imagawa, T., Watanabe, T. \& Nakamura, T. Subunit structure and multiple phosphorylation sites of phospholamban. J. Biochem. 99, 41-53 (1986).

24. Wegener, A. D. \& Jones, L. R. Phosphorylation-induced mobility shift in phospholamban in sodium dodecyl sulfate-polyacrylamide gels. Evidence for a protein structure consisting of multiple identical phosphorylatable subunits. J. Biol. Chem. 259, 1834-1841 (1984).

25. Colyer, J. Phosphorylation states of phospholamban. Ann. N. Y. Acad. Sci. 853, 79-91 (1998)

26. Anderson, M. E. Multiple downstream proarrhythmic targets for calmodulin kinase II: moving beyond an ion channel-centric focus. Cardiovasc. Res. 73, 657-666 (2007).

27. Federico, M., Valverde, C. A., Mattiazzi, A. \& Palomeque, J. Unbalance between sarcoplasmic reticulum $\mathrm{Ca} 2+$ uptake and release: a first step toward $\mathrm{Ca} 2+$ triggered arrhythmias and cardiac damage. Front. Physiol. 10, 2019.01630 (2020)

28. Chen, X. et al. Sorcin ablation plus beta-adrenergic stimulation generate an arrhythmogenic substrate in mouse ventricular myocytes. J. Mol. Cell. Cardiol. 114, 199-210 (2018).

29. Xu, M., Welling, A., Paparisto, S., Hofmann, F. \& Klugbauer, N. Enhanced expression of L-type Cav1.3 calcium channels in murine embryonic hearts from Cav1.2-deficient mice. J. Biol. Chem. 278, 40837-40841 (2003).

30. Cribbs, L. T-type calcium channel expression and function in the diseased heart. Channels 4, 447-452 (2010).

31. Kistamas, K. et al. Calcium handling defects and cardiac arrhythmia syndromes. Front. Pharm. 11, 72 (2020).

32. Maldonado, E. N. VDAC-tubulin, an anti-Warburg pro-oxidant switch. Front. Oncol. 7, 4 (2017).

33. Zhou, K. et al. VDAC2 interacts with PFKP to regulate glucose metabolism and phenotypic reprogramming of glioma stem cells. Cell Death Dis. 9, 988 (2018).

34. Chin, H. S. et al. VDAC2 enables BAX to mediate apoptosis and limit tumor development. Nat. Commun. 9, 4976 (2018).

35. Ren, D. et al. The VDAC2-BAK rheostat controls thymocyte survival. Sci. Signal. 2, ra48 (2009).

36. Harrington, J. L. \& Murphy, E. The mitochondrial calcium uniporter: mice can live and die without it. J. Mol. Cell. Cardiol. 78, 46-53 (2015).

37. Oka, T. et al. Cardiac-specific deletion of Gata4 reveals its requirement for hypertrophy, compensation, and myocyte viability. Circ. Res. 98, 837-845 (2006).

38. Drakos, S. G. et al. Impact of mechanical unloading on microvasculature and associated central remodeling features of the failing human heart. J. Am. Coll. Cardiol. 56, 382-391 (2010).

39. Warren, M. et al. Blockade of CaMKII depresses conduction preferentially in the right ventricular outflow tract and promotes ischemic ventricular fibrillation in the rabbit heart. Am. J. Physiol. Heart Circ. Physiol. 312, H752-H767 (2017).

40. Cluntun, A. A. et al. The pyruvate-lactate axis modulates cardiac hypertrophy and heart failure. Cell Metab. https://doi.org/10.1016/j.cmet.2020.12.003 (2020).

41. Sommakia, S. et al. Mitochondrial cardiomyopathies feature increased uptake and diminished efflux of mitochondrial calcium. J. Mol. Cell. Cardiol. 113, 22-32 (2017)

42. Ewels, P., Magnusson, M., Lundin, S. \& Kaller, M. MultiQC: summarize analysis results for multiple tools and samples in a single report. Bioinformatics 32, 3047-3048 (2016).

43. Love, M. I., Huber, W. \& Anders, S. Moderated estimation of fold change and dispersion for RNA-seq data with DESeq2. Genome Biol. 15, 550 (2014).

44. Kanehisa, M. \& Goto, S. KEGG: Kyoto Encyclopedia of Genes and Genomes. Nucleic Acids Res. 28, 27-30 (2000).
45. Muhlfeld, C. et al. Hypoinnervation is an early event in experimental myocardial remodelling induced by pressure overload. J. Anat. 222, 634-644 (2013).

46. Cerrone, M. et al. Bidirectional ventricular tachycardia and fibrillation elicited in a knock-in mouse model carrier of a mutation in the cardiac ryanodine receptor. Circ. Res. 96, e77-e82 (2005).

47. Sedej, S. et al. Subclinical abnormalities in sarcoplasmic reticulum $\mathrm{Ca}(2+)$ release promote eccentric myocardial remodeling and pump failure death in response to pressure overload. J. Am. Coll. Cardiol. 63, 1569-1579 (2014).

\section{Acknowledgements}

This work was supported by American Heart Association-Heart Failure Strategically Focused Research Network, Grant 16SFRN29020000 (Dr. Drakos); National Heart, Lung, and Blood Institute (NHLBI) RO1 Grant HL135121-01 (Dr. Drakos); NHLBI RO1 Grant HL132067-01A1 (Dr. Drakos). National Heart, Lung, and Blood Institute (NHLBI) R01 Grant GM071779 (Dr. Kwon). Additional support was provided by the Nora Eccles Treadwell Foundation to Dr. Drakos.

\section{Author contributions}

T.S.S. designed and performed experiments, analyzed data, and drafted the manuscript. D.K.A.R., K.S., S.S., R.B., A.T.K. and D. Ca performed experiments and helped to analyze the data. S.N. helped to analyze the data, edited the manuscript, and revised it critically for intellectual content. P.S., O.S.K., A.A., A.D. performed experiments, edited the manuscript, and revised it critically for intellectual content. J.L. and C.X. edited the manuscript and revised it critically for intellectual content. O.K. and C.X. synthesized efsevin and provided the required quantities to perform the requested experiments. K.S., P.S., A.D. and J.S. performed all experiments on efsevin, analyzed data, and edited the manuscript. O.K., E.H.Y.C., K.J.W., T.G., R.S.R., F.B.S., K.W.S., J.S. and D.Ch. helped with the data analysis, edited the manuscript, and revised it critically for intellectual content. S.G.D. provided overall guidance, conceived the research concept, supervised the study and data analysis, and critically revised the manuscript for intellectual content.

\section{Competing interests}

The authors declare no competing interests.

\section{Additional information}

Supplementary information The online version contains supplementary material available at https://doi.org/10.1038/s41467-021-24869-0.

Correspondence and requests for materials should be addressed to S.G.D.

Peer review information Nature Communications thanks Walter Koch and the other, anonymous, reviewer(s) for their contribution to the peer review of this work. Peer reviewer reports are available.

Reprints and permission information is available at http://www.nature.com/reprints

Publisher's note Springer Nature remains neutral with regard to jurisdictional claims in published maps and institutional affiliations.

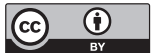

Open Access This article is licensed under a Creative Commons Attribution 4.0 International License, which permits use, sharing, adaptation, distribution and reproduction in any medium or format, as long as you give appropriate credit to the original author(s) and the source, provide a link to the Creative Commons license, and indicate if changes were made. The images or other third party material in this article are included in the article's Creative Commons license, unless indicated otherwise in a credit line to the material. If material is not included in the article's Creative Commons license and your intended use is not permitted by statutory regulation or exceeds the permitted use, you will need to obtain permission directly from the copyright holder. To view a copy of this license, visit http://creativecommons.org/ licenses/by/4.0/.

(C) The Author(s) 2021 